\title{
DTYMK is essential for genome integrity and neuronal survival
}

\author{
Jo M. Vanoevelen ${ }^{1,11}$ (1) . Jörgen Bierau ${ }^{1}$. Janine C. Grashorn ${ }^{1}$. Ellen Lambrichs ${ }^{1}$ - Erik-Jan Kamsteeg ${ }^{2}$. \\ Levinus A. Bok ${ }^{3}$. Ron A. Wevers ${ }^{4}$ - Marjo S. van der Knaap ${ }^{5} \cdot$ Marianna Bugiani $^{6} \cdot$ Junmei Hu Frisk $^{7} \cdot$ Rita Colnaghi $^{8}$. \\ Mark O'Driscoll $^{8}$ - Debby M. E. I. Hellebrekers ${ }^{1} \cdot$ Richard Rodenburg $^{4} \cdot$ Carlos R. Ferreira $^{9} \cdot$ Han G. Brunner $^{1,2,11,12,13}$. \\ Arthur van den Wijngaard ${ }^{1}$. Ghada M. H. Abdel-Salam ${ }^{10}$. Liya Wang ${ }^{7} \cdot$ Constance T. R. M. Stumpel $^{1}$
}

Received: 8 June 2021 / Revised: 3 December 2021 / Accepted: 3 December 2021 / Published online: 17 December 2021

(c) The Author(s) 2021

\begin{abstract}
Nucleotide metabolism is a complex pathway regulating crucial cellular processes such as nucleic acid synthesis, DNA repair and proliferation. This study shows that impairment of the biosynthesis of one of the building blocks of DNA, dTTP, causes a severe, early-onset neurodegenerative disease. Here, we describe two unrelated children with bi-allelic variants in $D T Y M K$, encoding dTMPK, which catalyzes the penultimate step in dTTP biosynthesis. The affected children show severe microcephaly and growth retardation with minimal neurodevelopment. Brain imaging revealed severe cerebral atrophy and disappearance of the basal ganglia. In cells of affected individuals, dTMPK enzyme activity was minimal, along with impaired DNA replication. In addition, we generated dtymk mutant zebrafish that replicate this phenotype of microcephaly, neuronal cell death and early lethality. An increase of ribonucleotide incorporation in the genome as well as impaired responses to DNA damage were observed in dtymk mutant zebrafish, providing novel pathophysiological insights. It is highly remarkable that this deficiency is viable as an essential component for DNA cannot be generated, since the metabolic pathway for dTTP synthesis is completely blocked. In summary, by combining genetic and biochemical approaches in multiple models we identified loss-of-function of DTYMK as the cause of a severe postnatal neurodegenerative disease and highlight the essential nature of dTTP synthesis in the maintenance of genome stability and neuronal survival.
\end{abstract}

Keywords DTYMK · dTMPK · Nucleotide metabolism · Zebrafish $\cdot$ Genome instability

Jo M. Vanoevelen and Jörgen Bierau are shared first authors.

Jo M. Vanoevelen

j.vanoevelen@maastrichtuniversity.nl

$\triangle$ Constance T. R. M. Stumpel

c.stumpel@mumc.nl

1 Department of Clinical Genetics, Maastricht University Medical Centre+, 6229 ER Maastricht, The Netherlands

2 Department of Human Genetics, Radboud UMC, 6525 GA Nijmegen, The Netherlands

3 Department of Pediatrics, Màxima Medical Center, 5504 DB Veldhoven, The Netherlands

4 Translational Metabolic Laboratory, Radboud UMC, 6525 GA Nijmegen, The Netherlands

5 Department of Child Neurology, VUMC, 1105 AZ Amsterdam, The Netherlands

6 Department of Neuropathology, VUMC, 1105 AZ Amsterdam, The Netherlands
Department of Anatomy, Physiology and Biochemistry, Swedish University of Agricultural Sciences, 75007 Uppsala, Sweden

8 Genome Damage and Stability Centre, University of Sussex, Brighton BN1 9RH, UK

9 National Human Genome Research Institute, National Institutes of Health, Bethesda, MD 20892, USA

10 Department of Clinical Genetics, Human Genetics and Genome Research Division, National Research Centre, Cairo 12311, Egypt

11 GROW-School for Oncology and Developmental Biology, 6229 ER Maastricht, The Netherlands

12 MHENS School of Neuroscience, 6229 ER Maastricht, The Netherlands

13 Donders Institute of Neuroscience, Radboud UMC, 6525 GA Nijmegen, The Netherlands 


\section{Introduction}

Cellular processes such as proliferation and differentiation, DNA and RNA synthesis, repair and catabolism require a tightly regulated nucleotide metabolism. One of the central components here is dTTP (deoxythymidine triphosphate). Thymidine nucleotides are biosynthesized by reutilization of thymine and thymidine known as the salvage pathway (Fig. 1a). Alternatively, dTMP (deoxythymidine monophosphate) is formed by the de novo pathway, in which dUMP (deoxyuridine monophosphate) is converted to dTMP by thymidylate synthase (Fig. 1a). The de novo biosynthetic pathway is considered the predominant pathway in actively replicating cells, whereas non-cycling cells rely on the salvage metabolism [3].

In this study, we describe a dramatic neurological condition in two unrelated children (Table 1). Exome analysis identified variants in DTYMK (deoxythymidine kinase) that segregate with this condition. Functional studies in affected fibroblasts and in zebrafish substantiated the evidence of a causal relationship. DTYMK encodes dTMPK (deoxythymidine monophosphate kinase), a key enzyme in dTTP nucleotide metabolism (Fig. 1a). The thymidine de novo and salvage pathways converge at the point of the phosphorylation of dTMP to dTDP by dTMPK [13]. This makes dTMPK the bottleneck for dTTP biosynthesis (Fig. 1a).

This report is the second study showing an association between DTYMK and a neurodegenerative condition [17]. In the current study, functional evidence and clues towards the pathophysiological mechanism are provided for the first time. We describe two individuals with pathogenic bi-allelic variants in DTYMK and detail the clinical and biochemical effects of these deleterious variants in the individuals and dtymk-deficient zebrafish.

\section{Materials and methods}

\section{Clinical samples, materials and exome sequencing}

DNA samples and skin biopsies were obtained from subjects in this study following written informed consent in a clinical genetic diagnostic setting. DNA was isolated from peripheral blood and fibroblasts were grown from skin biopsies. Photographs of affected individuals, used in this study were obtained after written approval from their legal guardians. Dermal fibroblasts were grown from punch skin biopsies in Minimal Essential Medium supplemented with L-glutamine, antibiotics (Pen-Strep) and $15 \%$ fetal bovine serum at $37{ }^{\circ} \mathrm{C}$ in a humidified atmosphere containing $5 \% \mathrm{CO}_{2}$.

Exomes were captured using the SureSelectXT Human All Exon $50 \mathrm{Mb}$ kit (Agilent Technologies) and sequenced on an Illumina HiSeq2000 by BGI-Europe (BGI Europe). Read mapping was performed using BWA (BurrowsWheeler Aligner) and variant calling using GATK (Genome Analysis ToolKit).

\section{Brain histology and immunohistochemistry}

The brain was fixed in buffered $4 \%$ formalin for 3 weeks and grossly sectioned. Tissue blocks were embedded in paraffin and cut at $5 \mu \mathrm{m}$ thickness. Tissue sections were stained with Hematoxylin and Eosin (H\&E), Klüver-periodic acid Schiff (Klüver-PAS) and Nissl according to routine methods. Immunohistochemistry was carried out as described [14] with antibodies against the astrocyte protein glial fibrillary acidic protein (GFAP; Millipore, 1:1000), the endothelium marker CD34 (DAKO, 1:200), the microglia marker Iba-1 (Wako, 1:10.000), the major mature myelin protein proteolipid protein (PLP; Serotec, 1:3000) and the apoptosis marker caspase3 (DAKO, 1:500). Positive and negative controls by omitting the primary antibody were included in each experiment. Briefly, sections were deparaffinized and rehydrated. Endogenous peroxidase activity was quenched by incubating the slides in $0.3 \%$ hydrogen peroxide in methanol. Slides were rinsed with distilled water and transferred to citric acid (pH6). Heat-induced antigen retrieval was performed using microwave irradiation for $15 \mathrm{~min}$ on low setting. Tissue sections were then cooled to room temperature and incubated overnight with primary antibodies. The antibody binding was visualized with 3, 3'-diaminobenzidine (DAB) staining. Sections were then counterstained with hematoxylin, mounted with polyvinyl alcohol medium with Dabco (Sigma) and photographed using a Leica DM6000B microscope (Leica Microsystems).

\section{Thymidylate kinase (dTMPK) assay}

In vitro dTMPK activity was determined as previously described [37]. Briefly, samples were thawed on ice in buffer A (320 mM sucrose, $5 \mathrm{mM}$ Tris pH 7.4, 2 mM EGTA (ethylene glycol-bis ( $\beta$-aminoethyl ether)-N, $\mathrm{N} \mathrm{N}^{\prime}, \mathrm{N}^{\prime}$-tetraacetic acid), Triton $\mathrm{X}-100,0.05 \%$ and protease inhibitors) and homogenized. Subsequently, homogenates were centrifuged at $16000 \times g$ for $15 \mathrm{~min}$ at $4{ }^{\circ} \mathrm{C}$ to pellet cellular debris. dTMPK activity was measured by incubating the protein extracts in a reaction mixture containing $50 \mathrm{mM}$ Tris $/ \mathrm{HCl}$, $\mathrm{pH} 7.6,0.5 \mathrm{mg} / \mathrm{ml}$ bovine serum albumin, $5 \mathrm{mM} \mathrm{MgCl}$, $5 \mathrm{mM}$ Dithiothreitol, $5 \mathrm{mM}$ ATP, $30 \mathrm{mM}$ Sodium fluoride (NaF), $0.1 \%$ Triton $\mathrm{X}-100$ and $25 \mu \mathrm{M}$ tritium-labeled dTMP $\left(\left[{ }^{3} \mathrm{H}\right]-\mathrm{dTMP}\right)$, which was prepared from $\left[{ }^{3} \mathrm{H}\right]$-thymidine using thymidine kinase catalyzed reaction. At $0,10,20$ and $30 \mathrm{~min}$., $10 \mu \mathrm{l}$ aliquots were removed and spotted onto Diethylaminoethyl cellulose (DEAE) filter paper (PerkinElmer) and dried. The filter papers were then washed in $50 \mathrm{mM}$ 
ammonium formate and sorted into scintillation vials. Reaction products were eluted with $0.5 \mathrm{ml}$ of $0.2 \mathrm{M} \mathrm{KCl}$ and $0.1 \mathrm{M} \mathrm{HCl}$, mixed with scintillation fluid and countered in a liquid scintillation counter (Tricarb, PerkinElmer).

\section{Zebrafish husbandry and genome editing}

Zebrafish (Danio rerio), AB strain, were housed in recirculating systems on a 14/10 day-night regime [18]. All animal studies were performed in concordance with the European and local guidelines and regulations of Maastricht University on animal experimentation.

Care of animals and animal experiments were conducted exclusively by licensed staff.

CrispR/Cas9-mediated genome editing was performed as described [10, 33]. A guideRNA (gRNA) targeting exon 4 (GGGACTTCCAAAACCAGACC) was designed using the CHOPCHOP tool [16]. The gRNA was in vitro translated using the MEGAShortscript T7-kit (ThermoFisher Scientific). Fertilized oocytes were injected with $100 \mathrm{ng} / \mu \mathrm{l}$ gRNA and $200 \mathrm{ng} / \mu \mathrm{l} \mathrm{Cas} 9$ mRNA (in vitro transcribed with the SP6 mMESSAGE mMACHINE kit (ThermoFisher Scientific) using the pCS2-nCas9n plasmid as a template) [10]. Genetically modified founders were identified by genotyping embryos from outcrosses using HRM (High-Resolution Melting curve analysis; see genotyping section) with primers HRMtmpk_exon4For and HRMtmpk_exon4Rev (Supplementary Table 1, online resource). Modification of the locus was confirmed using Sanger sequencing.

\section{Genotyping}

Sanger sequencing was performed on lysates of caudal fin biopsies of adult zebrafish, embryo lysates or purified DNA from human blood or fibroblasts. To this end, target regions were amplified by PCR and sequence analysis in both directions was performed using the ABI Big Dye Terminator Cycle Sequencing Ready Reaction kit and the ABI3730 Genetic Analyzer (Applied Biosystems). The primers used to genotype the human samples were: DTYMK_05_F and DTYMK_05_R. Genomic modification of the zebrafish dtymk locus was confirmed with M13-labeled primers zfdtymk_ex4For and zfdtymk_ex4Rev (Supplementary Table 1, online resource).

For high-resolution melting curve (HRM) analysis, zebrafish embryos were euthanized with a lethal dose of MS222 (tricaine). Biopsies from the caudal fin of adult fish were taken after anesthesia with MS222. Embryos or fin clips were placed individually in lysis buffer, consisting of $1 \mathrm{M} \mathrm{KCl}, 1 \mathrm{M} \mathrm{MgCl}_{2}, 1 \mathrm{M}$ Tris pH 8.3, Nonidet P40, Tween20 and gelatine, supplemented with Proteinase K $(100 \mu \mathrm{g} /$ $\mathrm{ml}$ ). Lysis was performed for $1 \mathrm{~h}$ at $60^{\circ} \mathrm{C}, 15 \mathrm{~min}$ at $95^{\circ} \mathrm{C}$ and then held at $4{ }^{\circ} \mathrm{C}$. After adding $80 \mu \mathrm{l}$ of water, lysates were used for PCR. Amplification and HRM analysis were performed in the LightCycler ${ }^{\circledR} 480$ (Roche Applied Science), using the HRM Master kit (Roche Applied Science) according to the following conditions: $95^{\circ} \mathrm{C}$ for $10 \mathrm{~min} ; 45$ cycles of $95{ }^{\circ} \mathrm{C}$ for $10 \mathrm{~s}, 59^{\circ} \mathrm{C}$ for $15 \mathrm{~s}, 72^{\circ} \mathrm{C}$ for $15 \mathrm{~s}$; one cycle of $95^{\circ} \mathrm{C}$ for $60 \mathrm{~s}, 40{ }^{\circ} \mathrm{C}$ for $60 \mathrm{~s}$, and a melting from $60{ }^{\circ} \mathrm{C}$ to $95{ }^{\circ} \mathrm{C}$ rising at $0.02{ }^{\circ} \mathrm{C}$ per second. All samples were run in triplicate. HRM analysis was performed using the software LightCycler ${ }^{\circledR} 480$ Gene Scanning version 1.5.1.

\section{Whole-mount antibody staining and histology}

Zebrafish embryos were fixed in $4 \%$ paraformaldehyde and dehydrated to $100 \%$ methanol and stored at $-20{ }^{\circ} \mathrm{C}$ until further processing. Prior to antibody staining, embryos were rehydrated to PBT (PBS $+0.1 \%$ Tween) and permeabilized using $10 \mu \mathrm{g} / \mathrm{ml}$ proteinase $\mathrm{K}$. After refixation in $4 \%$ paraformaldehyde, blocking was performed with $10 \%$ lamb serum, $0.1 \%$ Dimethyl sulfoxide (DMSO) for at least $3 \mathrm{~h}$ at room temperature. Next, the primary antibody was added for at least $2 \mathrm{~h}$. After extensive washing, the secondary antibody was added for at least 2 h. 3, 3'-diaminobenzidine (DAB)staining was performed using the liquid $\mathrm{DAB}+$ substrate chromogen system (Dako). Stained embryos were visualized and photographed using a stereomicroscope (Leica M165 FC). The antibodies used in this study were: anti$\gamma \mathrm{H} 2 \mathrm{AX}$ (gamma H2A histone family member X) (GeneTex GTX127342) and anti-phospho-Histone H3 (Ser10) (pH3) (Millipore 06-570).

For histological analysis, embryos were fixed in $4 \%$ paraformaldehyde and washed in phosphate buffered saline (PBS). Next, they were embedded in $2 \%$ low-melting point agarose to enable correct orientation for sectioning. Agarose blocks were trimmed, dehydrated and embedded in paraffin. $5 \mu \mathrm{m}$ sections were cut, air dried and stained with eosin/ hematoxylin. Sections were analyzed and photographed on a Nikon eclipse e800 microscope.

Apoptotic cells in zebrafish embryos were visualized using TUNEL (Terminal deoxynucleotidyl transferase dUTP nick end labeling) staining by means of the in Situ Cell Death Detection Kit (Roche) according to the manufacturers' instructions.

\section{Head size in larval zebrafish}

The size of the head was estimated by measuring the area between the eyes of embryos at 2 day post-fertilization (dpf) as described in Stankiewics et al. [32]. Briefly, pictures of the dorsal view of embryos were taken and the area of the region between the eyes was quantified using ImageJ software. 
a

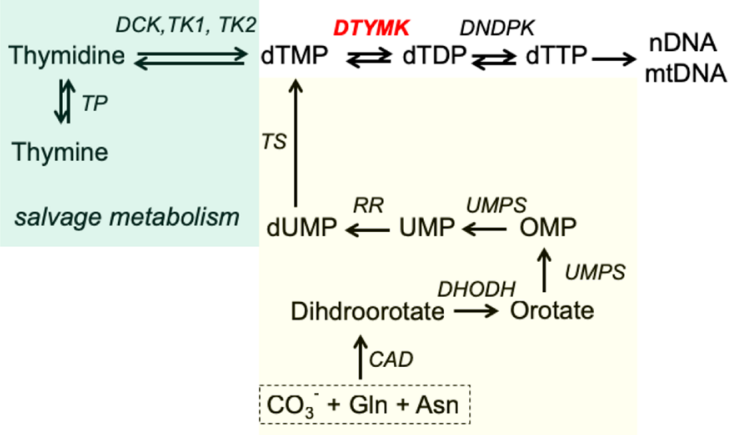

b

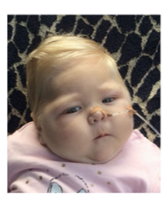

individual I

DTYMK: p.(Pro81Leu) DTYMK: p.(Asp128Asn)

\section{wt/mut} wt/mut

de novo synthesis

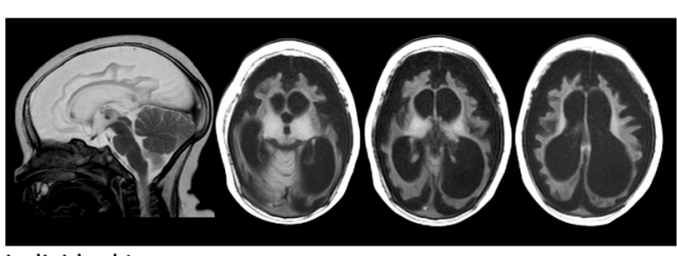

individual I

C
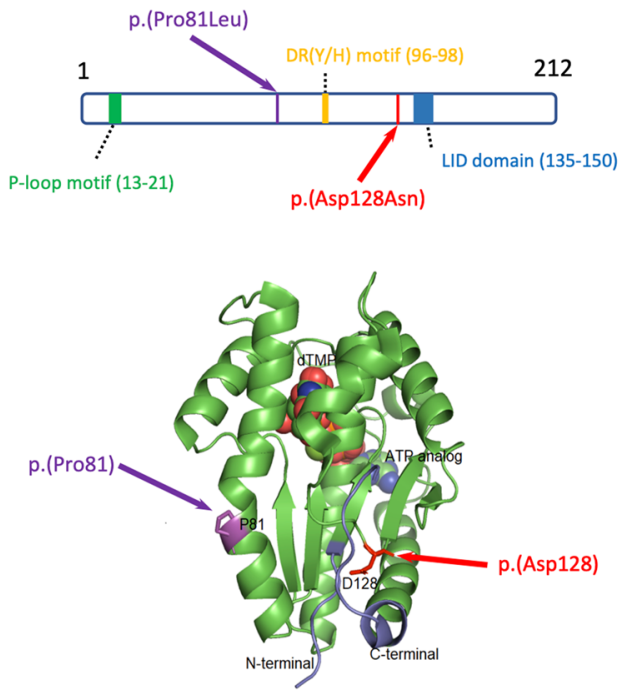

\section{DNA damage response signalling}

To assess the DNA damage response in vivo, zebrafish embryos at $24 \mathrm{hpf}$ (hours post-fertilization) were

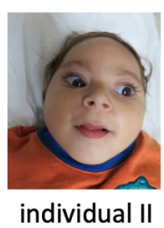

Family II

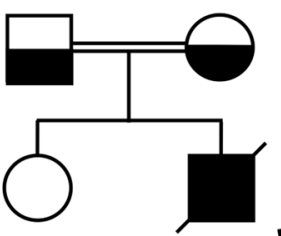

mut/mut

DTYMK: p.(Pro81Leu)

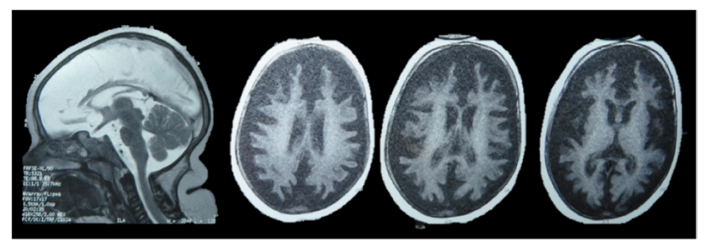

individual II

d

H.sapiens $\quad$...SVHLLFSANRWEQVPLIKEKLSQGVTLVVDRYAFSG... S.cerevisiae ...AIHLLFSANRWE IVDKIKKDLLEGKNIVMDRYVYSG... D.melanogaster ...VIHLMFSANRWEH INQVKEKLLEGTTLVVDRYSFSG.. D.rerio ...TVHLLFSANRWEMVPVMKQKLEEG INLVVDRYAFSG.. A.carolinensis $\quad$...TVHLLFSANRWEQVPLI KEKINQG I TLVVDRYAFSG.. $x$.tropicalis $\quad \ldots . .$. TIHLLFSANRWEQVPMI KEKIRSGVTLVIDRYAFSG. G.gallus M. Tusculus - SVHLLFSANRWEOVPLIKAKINOGVTLVIRYFG.

H.sapiens DVGLPKPDLVLFLOLOLADAAKRGAFGHERYENGAE... S.cerevisiae ...DVGLLKPDLTLFLSTODVDNNAEKSGFGDERYETVK.. D.melanogaster ...ERGLIKPDAVFYLRAPPNDLTHRGQYGKERYEKVEE... D.rerio ...DVGLPKPDLVMF LQLNPNVAANRGE YGNERYE TSAF... A.carolinensis $\quad$...DVGLPKPDVILFLQLNISEAAKRGDFGSERYENSSE... X.tropicalis $\quad$...DVGLPKPDLVLF LNVSPEVAASRGGFGNE I YE TNSE... $\begin{array}{ll}\text { G.gallus } & \text {...DAGLPKPDLI LFLQLSPEEAAARGNFGGERENGAF. } \\ \text { M.musculus } & \text {...DVGLPKPDLILFLQLQLLDAAARGEFGLERYETGTE.. }\end{array}$

UV-irradiated in a UV Stratalinker 1800 (Stratagene) at a dose of 12,000 microjoule $/ \mathrm{cm}^{2}$. Subsequently, they were left to recover for $24 \mathrm{~h}$ at $28^{\circ} \mathrm{C}$ and processed for antibody staining with the anti- $\gamma \mathrm{H} 2 \mathrm{AX}$ antibody. 
४Fig. 1 Description of variants and clinical phenotype. a Overview of the dTTP biosynthesis pathway. DTYMK (dTMPK) mediates the penultimate step of the generation of dTTP. Both pathways: salvage pathway and de novo pathway converge before the enzymatic defect in DTYMK. Abbreviations used: $D C K$ deoxycytidine kinase; $T K 1$ thymidine kinase 1; TK2 thymidine kinase 2 (mitochondrial); $D T Y M K$ deoxythymidine (monophosphate) kinase; $D N D P K$ deoxynucleotide diphosphate kinase; $T S$ thymidylate synthase; $R R$ ribonucleotide reductase; UMPS uridine monophosphate synthetase; $C A D$ carbamoyl-phosphate synthase 2, aspartate transcarbamylase and dihydroorotase; TP thymidine phosphorylase. b Description of two families carrying variants in $D T Y M K$. Both families share the variant c. $242 \mathrm{C}>\mathrm{T}$ which is homozygous in individual II and indicated in purple. Family I is compound heterozygous and contains the additional variant c. $382 \mathrm{G}>\mathrm{A}$, indicated in red. Brain MRI and pictures showing microcephaly in both affected individuals. The age of the individuals at which the pictures were taken are 9 months and 26 months for individual I and individual II, respectively. MRI images of individual I at age 6 months and of individual II at 12 months. Both subjects show severe atrophy of the cerebral hemispheres and basal ganglia, whereas the thalamus, brain stem and cerebellum appear normal. Individual I also shows pronounced dilation of the lateral ventricles. c Position of the observed variants in the primary protein sequence of dTMPK and relative position of known functional domains are shown in the upper panel. The variants are labelled in purple (p.(Pro81Leu)) and red (p.Asp128Asn). Known functional domains of DTYMK are: P-loop motif (indicated in green, residues 13-21), DR(Y/H)-motif (yellow, residues 96-98), LID domain (blue, residues 135-150) [26]. In the lower panel, the position of the variants in the 3D structure (PDB code 1e2f) are depicted in purple (p.Pro81Leu) and red (p.Asp128Asn). d Evolutionary conservation of regions in dTMPK containing the described variants. The protein sequences used in the alignment are: H.sapiens: ENSP00000304802; S. cerevisiae: YJR057W;D. melanogaster: FBpp0303030; D. rerio: ENSDARP00000068373; A. carolinensis: ENSACAP00000000660; X. tropicalis: ENSXETP00000044270; G. gallus: ENSGALP00000053279; M. musculus: ENSMUSP00000027503

\section{EdU labeling}

Fibroblasts were labelled with $10 \mu \mathrm{M}$ EDU (5-ethynyl-2'-deoxyuridine) for $30 \mathrm{~min}$, then processed using Click-IT technology as described by the manufacturer (ThermoFisher Scientific). Subsequently, cells were analyzed in a BD Accuri C6 Plus flow cytometer. Profiles were processed using BD CSampler software (BD Biosciences).

\section{Alkaline gel electrophoresis}

Incorporation of ribonucleotides was investigated using alkaline agarose gel electrophoresis as described in Sambrook et al. [31]. Genomic DNA was isolated using Qiagen Blood and Tissue kit and incubated at $55{ }^{\circ} \mathrm{C}$ for $2 \mathrm{~h}$ in the presence of $300 \mathrm{mM} \mathrm{NaOH}$ before electrophoresis on alkaline agarose gels. DNA was visualized by ethidium bromide staining or SYBR Gold staining (Thermo Fisher).

\section{Mitochondrial measurements}

Mitochondrial DNA (mtDNA) copy number was measured in genomic DNA preparations. mtDNA copy number was assessed by comparing expression levels of a mitochondrial encoded gene $(N D 1)$ versus a nuclear encoded gene $(B 2 M)$. Relative mtDNA copy number was calculated by calculating $\Delta \mathrm{CT}$ (=CT nuclear gene, B2M-CT mitochondrial gene, ND1) and converting $\Delta \mathrm{CT}$ to relative expression levels (relative expression $=2^{\Delta C T}$ ) as described in Rahn et al. [27].

The integrity of the mitochondrial genome was assessed by PCR amplification of the complete mitochondrial genome by long-range PCR using Phusion Hot Start polymerase II using primers MM_16426_NGS_F and MM16425_NGS_R (Supplementary Table 1, online resource), followed by agarose gel electrophoresis.

Complex activity in fibroblasts of individual I and parents was analyzed as follows: fibroblasts were cultured and harvested for enzyme analysis. Complex activities were determined as described by Rodenburg and coworkers [29]. This assay is a diagnostic assay, performed at the Translational Metabolic Laboratory of the Radboud UMC.

\section{Statistical analysis}

Statistical evaluation and data presentation were performed using GraphPad Prism 8 software. Microsoft Excel was used to calculate the exact $p$ values. Statistical significance was calculated using the Student's $t$ test (unpaired, two-sided). Differences were considered statistically different when $p<0.05$. Variance is calculated when comparing different groups and was equal unless indicated otherwise.

\section{Results}

1. Clinical phenotypes

\section{Individual I}

The proband was the first child of unrelated Dutch parents (Fig. 1b). Family history was uneventful with no prior miscarriages. Pregnancy was induced by in vitro fertilization because of male subfertility and was complicated by polyhydramnios. Third-line ultrasound examinations at 30th and 36th weeks of gestation, however, showed no explanation for the polyhydramnios. No congenital abnormalities were observed. Femur length growth was at the 10th centile. The fetal brain looked normal and the fetus had a normal fetal head circumference, also at the 10th centile (Table 1). There was no known teratogenic exposure.

Delivery was uncomplicated at 37 weeks of gestation. Birthweight was $2645 \mathrm{~g}$ (15th centile Fenton growth chart 
Table 1 Overview of the most important clinical features of individual I and individual II

\begin{tabular}{|c|c|c|}
\hline & Patient I & Patient II \\
\hline \multirow[t]{2}{*}{ Variant, genomic } & Maternal: Chr2(GRCh37):g.242618013C $>$ T & Maternal: Chr2(GRCh37):g.242619732G >A \\
\hline & Paternal: Chr2(GRCh37):g.242619732G >A & Paternal: Chr2(GRCh37):g.242619732G>A \\
\hline \multirow[t]{2}{*}{ Variant, cDNA } & Maternal: NM_012145.3:c.382G>A & NM_012145.3:c.242C $>\mathrm{T}$ \\
\hline & Paternal: NM_012145.3:c.242C $>$ T & \\
\hline Variant, protein & Maternal: p.Asp128Asn; Paternal: p.Pro81Leu & p.Pro81Leu \\
\hline Origin & The Netherlands & Egypt \\
\hline Prenatal findings & Polyhydramnios, OFC at 36 weeks 10 th centile & Slightly smaller head \\
\hline Microcephaly & Yes & Yes \\
\hline Development & No developmental progress & No developmental progress \\
\hline Hearing & Normal & Normal \\
\hline Vision & No tracking of objects, only light/dark & No tracking of objects \\
\hline Sex & Female & Male \\
\hline Birth weight, grams / SD & $2645 /-2.3 \mathrm{SD}$ & $2250 /-1.8 \mathrm{SD}$ \\
\hline Seizures & Recurrent febrile seizures & myoclonic jerks \\
\hline Weight, kg / age, months / SD & $8,0 / 17 /-2.6 \mathrm{SD}$ & $7.2 / 30 /-4.0 \mathrm{SD}$ \\
\hline Height, cm / age, months / SD & $58 / 16 /-6.5 \mathrm{SD}$ & $68 / 26 /-5.4 \mathrm{SD}$ \\
\hline $\begin{array}{l}\text { Head circumference, } \mathrm{cm} / \text { age, } \\
\text { months, / SD }\end{array}$ & $34 / 9 /-7.6 \mathrm{SD}$ & $38 / 26 /-7.4 \mathrm{SD}$ \\
\hline \multirow[t]{2}{*}{ MRI brain } & $\begin{array}{l}\text { Severe atrophy of cerebral hemispheres and basal } \\
\text { ganglia }\end{array}$ & Profound generalized volume loss cerebral cortex \\
\hline & Brain stem and cerebellum appeared normal & Cerebellum unaffected \\
\hline \multirow[t]{4}{*}{ Other findings } & Puffy body & puffy hands and feet, full cheeks \\
\hline & Died at age 18 months & Died at age 32 months \\
\hline & & $\begin{array}{l}\text { Microcytic hypochromic anemia and elevated liver } \\
\text { enzymes }\end{array}$ \\
\hline & & Micropenis and undescended testis \\
\hline
\end{tabular}

for girls 37 weeks of gestation; 25th centile Perined), occipitofrontal circumference (OFC) was $31 \mathrm{~cm}$ ( 2 nd centile) and height was $44 \mathrm{~cm}$ (10th centile); Apgar scores were 9/10 after 1 and $5 \mathrm{~min}$, respectively. No apparent dysmorphic features were observed (Table 1). Because of poor feeding, she was readmitted to the hospital on the 3rd day. Nutrition with naso-gastric tube was needed and well tolerated. However, normal growth was never achieved. The OFC and height progressively deviated from the normal centiles. She developed severe microcephaly with a very puffy appearance (Fig. 1b). The girl was hypotonic at birth but developed spasticity with opisthotonus within 1 year of age. No neurodevelopmental milestones were achieved. No eye contact was ever made. She developed recurrent febrile seizures with flat trace EEG at 6 months of age which were successfully treated with phenobarbital.

Brain MRI at age 3 weeks of life showed slight underdevelopment of the caudate nucleus and the frontal cortex, with severe underdevelopment of the putamen. Brain MRI at 6 months of age showed dramatic atrophy of the cerebral hemispheres with severe enlargement of the lateral ventricles and subarachnoid spaces (Fig. 1b, individual I). At the basal ganglia, only the thalamus appeared of normal volume. On MRI, the brain stem and cerebellum also appeared normal (Fig. 1b, individual I). Microarray showed a normal karyotype: $46, \mathrm{XX}$.

Extensive metabolic investigations were performed including urinary organic acids, amino acids, purines \& pyrimidines, glycosaminoglycans, oligosaccharides, acylglycines, creatine and guanidinoacetate, bile acids and bile alcohols. Plasma amino acids, acylcarnitines, very-long chain fatty acids, sterols, chitotriosidase, creatine and guanidinoacetate, sterols, and plasmalogens were normal. CSF amino acids were without abnormalities. At 18 months of age she had a respiratory illness. This was complicated by cardio-pulmonary arrest, leading to demise.

Post-mortem examination of the internal organs showed no abnormalities. Gross neuropathological examination showed severe atrophy of the neocortex, cerebral white matter and basal ganglia, with normal size of brainstem and cerebellum (Fig. 2 Overview). Microscopy of the cerebral cortical ribbon revealed massive loss of neurons with astrogliosis and minimal vascular proliferation (Fig. 2 Histology). Whole mounts of the cerebral hemispheres at the level of the 
Fig. 2 Neuropathology. Overview (upper) Panel. Macroscopic aspect of the brain of individual I. (a, b) Coronal cut through the cerebral hemispheres $(\mathbf{a}, \mathbf{b})$, cerebellum (a) and brainstem (c, pons on the left, medulla oblongata on the right). Histology (lower) panel. Microscopic aspect of the brain of individual I. a Whole mounts of the cerebral hemispheres confirm the massive degree of global atrophy (a, haematoxylin and eosin, HE) and show paucity of myelin throughout the cerebral hemispheres and capsules (Kluver-PAS). b-e Photomicrographs of the cerebral cortex from the frontal lobe show loss of neurons, including pyramidal cells (b, HE; c, Nissl stain for nuclei), astrogliosis (d, glial fibrillary acidic protein, GFAP) and minimal degree of vascular proliferation (e, CD34). f, $\mathbf{g}$ Photomicrographs of the deep white matter adjacent to the lateral ventricles show loss of oligodendrocytes (f, HE) and moderate activation of microglia (IBA-1). h-j The more peripheral hemispheric white matter shows massive activation of microglia with ameboid morphology (h, IBA-1), lack of myelin (i, proteolipid protein stain for myelin) and loss of oligodendrocytes by apoptosis (j, CASP3 stain for apoptotic cells). k, I Photomicrographs of the basal ganglia (k, HE) and thalamus (l, Nissl) show loss of neurons and reactive gliosis. $\mathbf{m}, \mathbf{n}$ In the cerebellum, microscopic examination shows loss of neurons in the granular and Purkinje cell layer (m, HE) and moderate reaction of the Bergmann glia (n, GFAP)

\section{Overview}

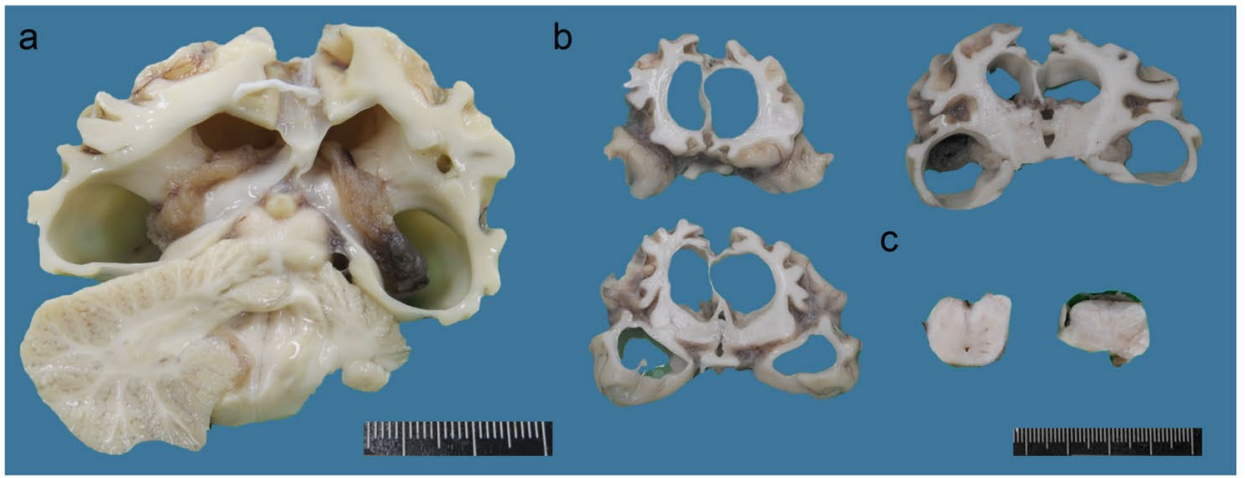

Histology

a

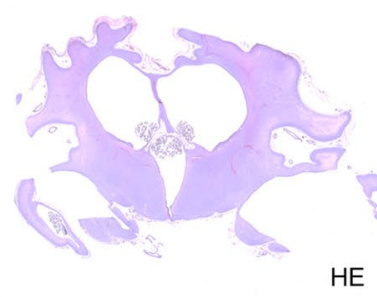

HE

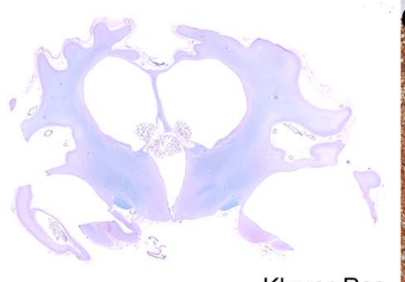

Kluver-Pas
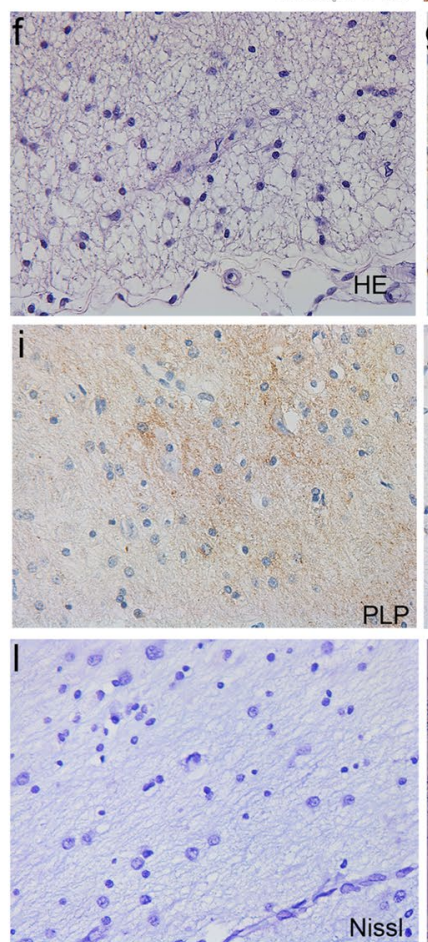
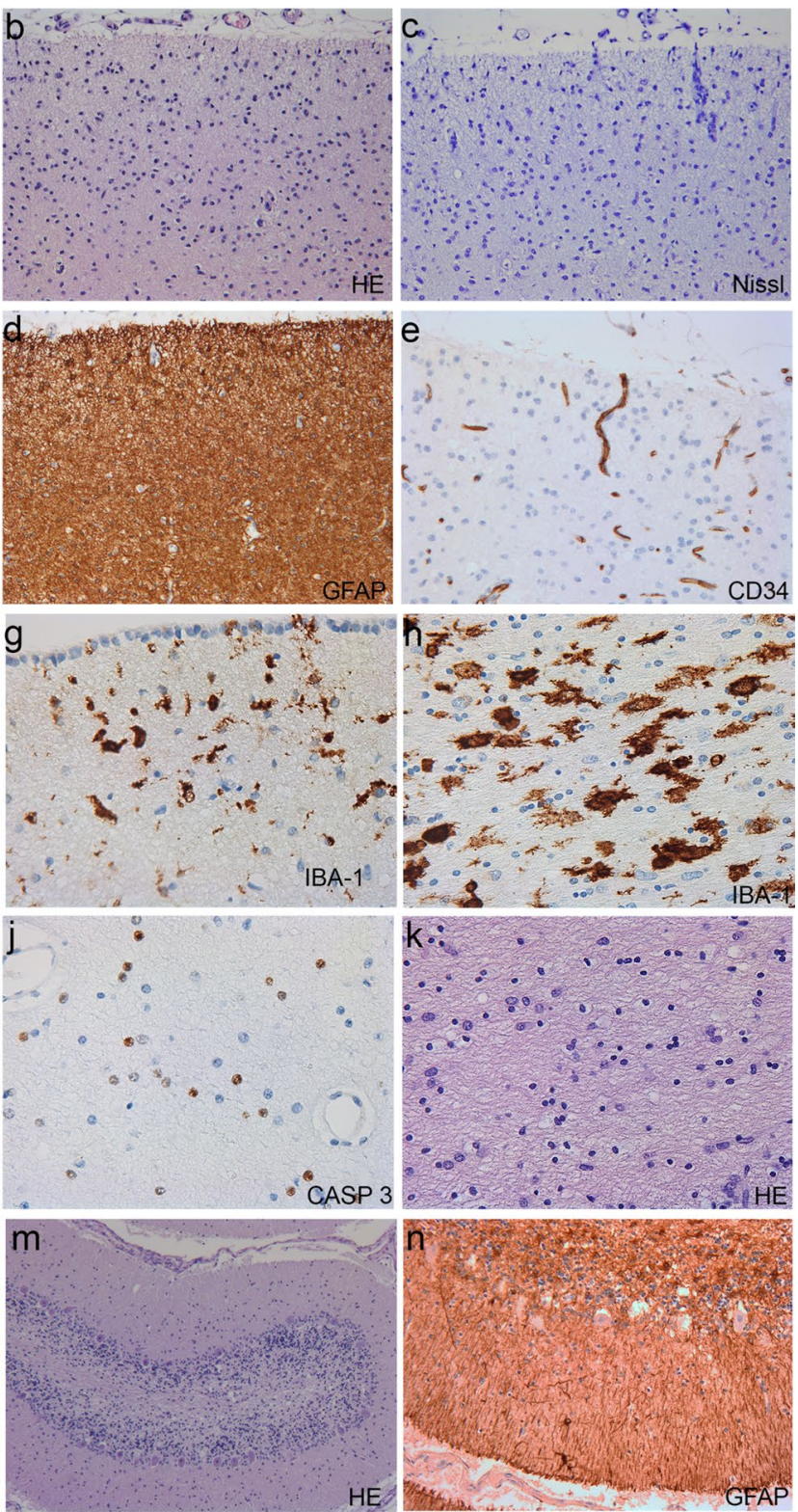
neostriatum confirm the massive degree of global atrophy (Fig. 2a, haematoxylin \& eosin, HE) and show paucity of myelin throughout the cerebral hemispheres and capsules (Fig. 2a, Kluver-PAS). Photomicrographs of the cerebral cortex from the frontal lobe show loss of neurons, including pyramidal cells (Fig. 2b, HE; c, Nissl stain for nuclei), intense anisomorphic astrogliosis (Fig. 2d) and minimal degree of vascular proliferation (Fig. 2e). Photomicrographs of the deep white matter adjacent to the lateral ventricles show loss of oligodendrocytes (Fig. 2f, HE) and moderate activation of microglia (Fig. 2g). The more peripheral hemispheric white matter shows massive activation of microglia with ameboid morphology (Fig. 2h), lack of myelin (Fig. 2i) and loss of oligodendrocytes by apoptosis (Fig. 2j). Photomicrographs of the neostriatum (Fig. 2k, HE) and thalamus (Fig. 21, Nissl) show loss of neurons and reactive gliosis. In the cerebellum (Fig. $2 \mathrm{~m}, \mathrm{n}$ ), microscopic examination shows loss of neurons in the granular and Purkinje cell layer (Fig. 2m, HE) and moderate reaction of the Bergmann glia (Fig. 2n, GFAP).

\section{Individual II}

This boy was the second child of healthy consanguineous Egyptian parents (first cousins, Fig. 1b). There is a healthy older sister. Pregnancy was uneventful, with no known teratogenic exposure. At the 32nd week of gestation, the prenatal ultrasound showed smaller biparietal diameter for gestational age. The individual was born at term by elective cesarean. His birth weight was $2250 \mathrm{~g}(-1.8 \mathrm{SD})$ (Table 1). His birth height and OFC were not documented but the latter was noticed to be small. He stayed in the neonatal intensive care unit for 7 days because of neonatal jaundice that was treated with phototherapy. The individuals' parents became concerned during the first months of life as he did not offer eye contact or track objects. At the age of 7 months, he presented with fever and repeated vomiting and was admitted to the hospital. Severe anemia was detected and treated by blood transfusion. The individual underwent multiple analyses of hematological indices and liver enzymes at different occasions that always showed microcytic hypochromic anemia and elevated liver enzymes, respectively.

He developed severe myoclonic jerks at 15 months of age, which were treated with carbamazepine. The EEG demonstrated slow background activity and occasional sharp waves. Metabolic work-up including glucose, lactate, ammonia, biotinidase, creatine kinase, acylcarnitines, amino acids, very long-chain fatty acids and urinary organic acids profile were all normal.

At 30 months of age, the boy was referred for genetic counseling. On examination, his weight was $7200 \mathrm{~g}(-4$ SD), height was $68 \mathrm{~cm}(-5.4 \mathrm{SD})$, and $\mathrm{OFC}$ was $38 \mathrm{~cm}$ (- 7.4 SD) (Table 1). He had fairer hair and skin compared to his parents and sister. Bitemporal narrowing, full cheeks, puffy hands and feet were noted (Fig. 1b, individual II). Furthermore, he had bilateral undescended testes and micropenis. There was good control of the head and increased tone distally in the arms and legs. Tendon reflexes were increased, with bilateral clonus and positive Babiniski signs. He made almost no developmental progress: he did not roll over, sit, vocalize, or smile. Ophthalmologic examination and hearing test were normal. Serum electrolytes, urea, creatine, albumin level and 17-hydroxyprogesterone were all normal. Chromosome analysis showed a normal 46, XY karyotype. Brain MRI and CT at age 2 years showed profound generalized cerebral atrophy with widening of the subarachnoid spaces (Fig. 1b, individual II). The basal nuclei were small, but visible, while the thalamus appeared to have a normal size. Also, the brain stem and cerebellum were spared (Fig. 1b, individual II). At the age of 32 months, the boy developed a pneumonia followed by coma and death.

At birth, no apparent dysmorphic features were observed in both children. In the months following birth, the following phenotypic features became apparent in both individuals: growth retardation, puffy body, seizures, failure to reach developmental milestones, microcephaly and severe, progressive atrophy of the cerebral hemispheres and basal ganglia (Table 1). Pathology in individual I confirms massive neuronal dropout, only sparing the dentate nucleus and brain stem.

\section{Description of variants in DTYMK}

Exome sequencing identified compound heterozygous variants in DTYMK (Deoxy Thymidylate Kinase) in family I: Chr2(GRCh37):g242619732G $>$ A; NM_012145.3:c.242C>T; p.Pro81Leu (paternal) and Chr2(GRCh37):g242618013C $>$ T; NM_012145.3:c.382G>A; p.Asp128Asn (maternal) (Fig. 1b; Table 1). In the second case (individual II), one of the abovementioned variants, Chr2(GRCh37):g242619732 G>A;NM_012145.3:c.242C>T; p.Pro81Leu was observed in a homozygous state. (Fig. 1b; Table 1).

Variant c242C $>$ T, p.(Pro81Leu) is a missense substitution of a highly conserved nucleotide (phyloP: 5.29 [ -14.1 ; 6.4]) leading to a change in a moderately conserved amino acid (considering 13 species) (Fig. 1d). Predictions of the amino acid change, p.(Pro81Leu), varied from tolerated (SIFT, score: 0.19, median: 2.82) to "possibly damaging" (PolyPhen2 score 0.885) to disease causing (MutationTaster, $p$ value: 1$)$. This variant is unknown to ESP and ExAC. In dbSNP, it is known as rs 1267106442 and is found once in gnomAD with a MAF of 0.0000319 , but only in a heterozygous state (accessed on 04/05/2021) (Supplementary Fig. 2a, online resource). 
The second variant, c.382G $>$ A, p.(Asp128Asn) is also a missense substitution involving a highly conserved nucleotide (phyloP: $5.61[-14.1 ; 6.4]$ ), giving rise to a change in a highly conserved amino acid, up to Saccharomyces cerevisiae (considering 13 species) (Fig. 1c, d). SIFT-prediction was tolerated (score: 0.09, median: 2.82), whereas MutationTaster and Polyphen-2 predicted this variant to be diseasecausing ( $p$ value: 1$)$ and probably damaging (score 1.00), respectively (Supplementary Fig. 2a, online resource). This variant is known to bSNP as rs373875797 (MAF/MinorAlleleCount: $\mathrm{T}=0.000 / 0)$ and to ESP as ESP6500SIV2. This variant has been reported twice in the ExAC database (MAF: $0.00001649)$, but never in a homozygous state. This variant is known to gnomAD and appears 4 times with a MAF of 0.00001591 , but only in a heterozygous state (accessed on 04/05/2021). Both variants are not located in a known functional domain of the protein (Fig. 1c).

\section{Zebrafish dtymk knockout model}

To study the relationship between neurodegeneration and dTMPK deficiency, we generated a dtymk knockout model in zebrafish (Fig. 3). Sequencing of homozygous mutant embryos revealed a 5-bp deletion in exon 4 (Fig. 3a). This modification leads to a premature stop codon, 19 amino acids downstream of the deletion, whereas the wildtype protein contains another 85 amino acids. The first 127 amino acids $(60 \%)$ of the tmpk protein upstream remain unaltered (Fig. 3a).

At $5 \mathrm{dpf}$, all homozygous dtymk mutant embryos show small eyes, pericardial edema and edema around the intestine, while $2 / 3$ of the mutant embryos also had extensive edema of the brain (Fig. 3b; Supplementary Fig. 2b, online resource). The fraction of affected embryos was consistent with the expected fraction from a heterozygous incross (25\%), and confirmed by genotyping (Supplementary Fig. 2b, online resource). From 3dpf onwards, dtymk mutant embryos can be morphologically distinguished from their siblings as the eyes are markedly smaller and pericardiac edema is prominent. Remarkably, mutant embryos at $3 \mathrm{dpf}$ show twitching movements, reminiscent of epileptic seizures although other causes (eg. motor neuron dysfunction) cannot be excluded. From 4dpf onwards, mutant embryos develop prominent edema of the brain and around the intestine. At 5dpf, more than $40 \%$ of mutant embryos have died. Since affected individuals show microcephaly, the head size of mutant and sibling embryos was quantified at $2 \mathrm{dpf}$ (Fig. 3d). A timepoint early in development was chosen to avoid brain edema interfering with the assessment of head size. Head size was found to be significantly $\left(p=1.52 \times 10^{-13}\right)$ smaller when compared to sibling embryos of the same batch (Fig. 3d). The length of sibling vs. mutant embryos was not different (Supplementary Fig. 1c), ruling out developmental delay as the cause of microcephaly in dtymk mutant zebrafish.

Histology of 3- and 5-day-old embryos provided a more detailed insight into the phenotype (Fig. 3c). In the brain, empty spaces, indicative of neurodegeneration are apparent in the mutant embryos (Fig. 3c, blue star). In addition, cartilage structures of the lower jaw (Meckels' cartilage) are absent in the mutant larvae (Fig. 3c blue arrows). Severe underdevelopment of the eyes is prominent: the eyes of mutant embryos are smaller, have an irregular shape and only show the retinal pigmented epithelium (RPE) and photoreceptor layer (PRL), whereas the other 4 layers are absent (Supplementary Fig. 2d, online resource).

TUNEL staining at $2 \mathrm{dpf}$ revealed higher amounts of apoptotic cells in dtymk mutant zebrafish. We quantified the number of TUNEL-positive cells in the forebrain of dtymk mutant zebrafish and found that dtymk mutant embryos have significantly more apoptotic cells in the forebrain when compared to wildtype $\left(p=6.45 \times 10^{-6}\right)$ or heterozygous $\left(p=7.54 \times 10^{-6}\right)$ siblings (Fig. $\left.4 \mathrm{e}, \mathrm{f}\right)$.

These results, together with the observation of extensive postnatal cerebral atrophy in both individuals and the presence of apoptotic oligodendrocytes in the brain of individual I (Fig. 2j) demonstrate that neuronal cell death is a hallmark of the disease phenotype as it is prominent in both the affected individual as the zebrafish model as a result of dtymk deficiency.

4. $D T Y M K$ alleles in affected individuals and zebrafish are loss-of-function

The functional effect of the variants, observed in the individuals and the deletion in the zebrafish model were assessed at the biochemical level using an in vitro dTMPK assay [37]. To this end, dTMPK activity was measured in cultured fibroblasts of family I (Fig. 4a). In fibroblasts of the parents, dTMPK activity was readily detectable. In the mother, an activity of $43.65 \mathrm{pmol} / \mathrm{min} / \mathrm{mg}$ protein was measured, whereas the cells of the father showed lower activity: $31.08 \mathrm{pmol} / \mathrm{min} / \mathrm{mg}$ protein. dTMPK activity of the individual was hardly detectable: $0.62 \mathrm{pmol} / \mathrm{min} / \mathrm{mg}$ protein. dTMPK enzyme activity of the individual is significantly lower than those of the parents (mother vs. individual I $p=1.46 \times 10^{-6}$; father vs. individual $p=5.77 \times 10^{-6}$ ).

In the zebrafish model, pools of 35-50 larvae of 5dpf were phenotyped and processed for dTMPK enzyme activity measurements (Fig. 4b). The sibling embryos had a dtmpk activity of $38.55 \mathrm{pmol} / \mathrm{min} / \mathrm{mg}$ protein, which is comparable to the activity measured in the fibroblasts of the parents. Dtymk mutant embryos on the other hand had barely detectable activity: $1.80 \mathrm{pmol} / \mathrm{min} / \mathrm{mg}$ protein. Unrelated wildtype larvae of the same age showed a dtmpk activity of $41.43 \mathrm{pmol} / \mathrm{min} / \mathrm{mg}$ protein. Dtmpk activity of mutant 

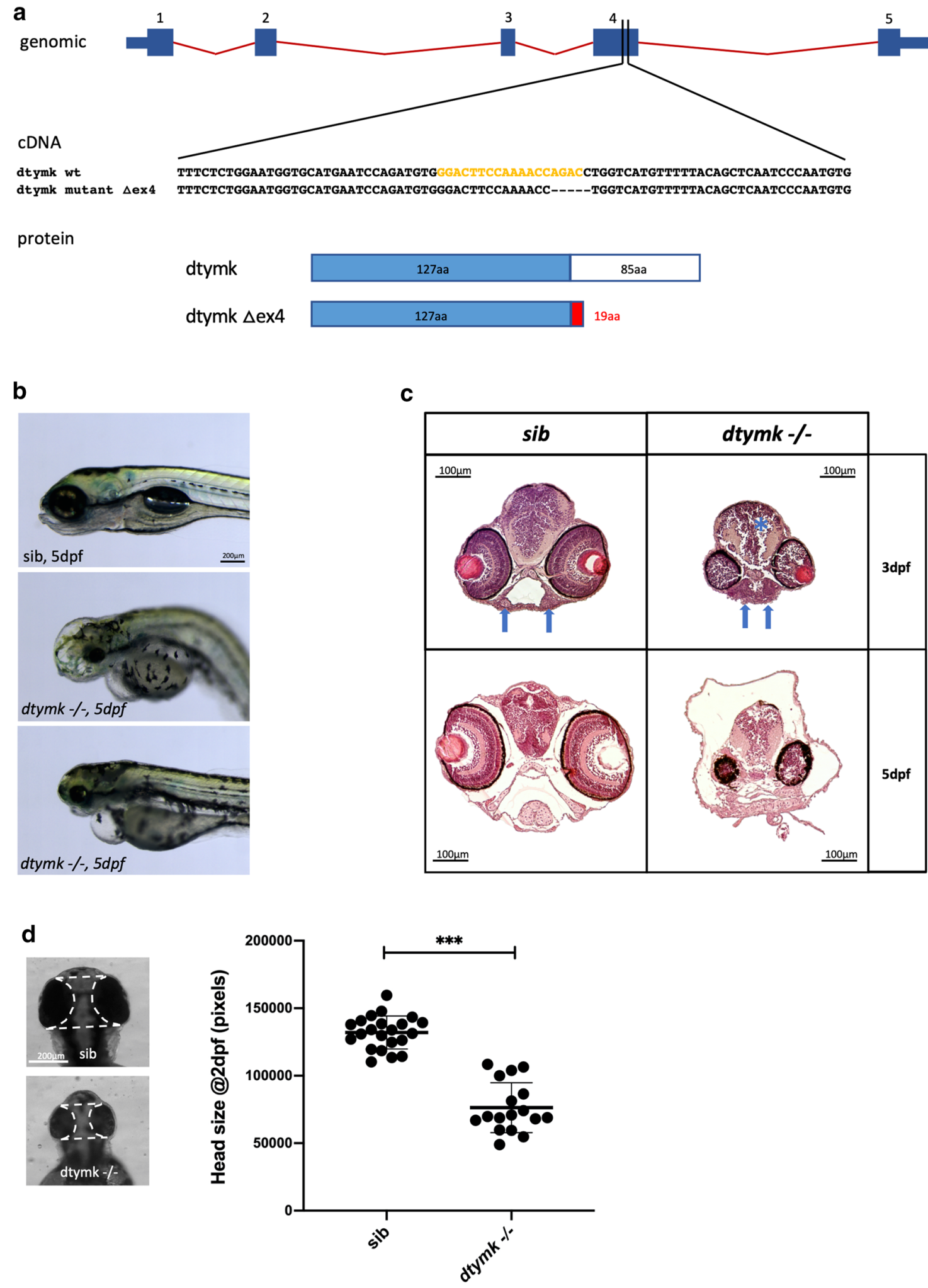

larvae is significantly lower than those of sibling larvae $(p=0.0028)$ or wildtype larvae $(p=0.0001)$ (Fig. $4 b)$.

Similar results from fibroblasts of an affected individual and knockout zebrafish confirm that the tested alleles in fibroblast and zebrafish represent loss-of-function alleles, giving rise to a non-functional dTMPK enzyme, shown by the very low dTMPK activity in both models. 
4Fig. 3 dtymk knockout zebrafish. a Genomic location of the gRNA sequence used to generate the knockout line of dtymk, indicated in yellow. The mutant line, $\Delta$ ex 4 contains a 5-bp deletion in exon 4 resulting in a truncated protein. b Phenotype of dtymk knockout (dtymk - -) embryos at 5dpf and their siblings (sib). The homozygous mutant embryos (dtymk -/-) show small, irregular eyes and edema around the brain, heart and intestine. The scale bar represents $200 \mu \mathrm{m}$. c Hematoxylin/eosin staining of transversal sections through the head of sibling and dtymk mutant embryos at $3 \mathrm{dpf}$ and $5 \mathrm{dpf}$ at the level, where the optic nerve exits the eye. Images show prominent degeneration of brain tissue (blue star) and structural abnormalities of the eyes and jaw cartilage structures (blue arrow) in the mutant embryos (dtymk -/-) which is absent in the sibling (sib) embryos. The scale bar represents $100 \mu \mathrm{m}$. d Quantification of head size in dtymk -/- and sibling (sib) embryos at 2dpf. Average head size of 19-24 embryos at $2 \mathrm{dpf}$ showing significant microcephaly in mutant vs. sibling embryos (***, $p=1.52 \times 10^{-13}$ ). Error bars show the standard deviation. Two biological replicates. The scale bar represents $200 \mu \mathrm{m}$

5. DTYMK disease alleles cause impaired DNA replication, elevated ribonucleotide incorporation and altered DNA damage signaling

Since the effect of the variants in DTYMK completely blocks the known biosynthesis pathway of dTTP, we evaluated processes that require significant amounts of nucleotides including DNA replication and aspects of DNA repair. To assess DNA replication, we performed pulse-EdU staining of fibroblasts from individual I and the parents to quantify the number of cells in S-phase of the cell cycle (Fig. 4c). Flow cytometry analysis of EdUlabeled fibroblasts showed that in the parents of family I, the number of S-phase cells is $23.65 \%$ and $16.03 \%$ in the mother and father, respectively. In contrast, the fraction of S-phase cells in individual I fibroblasts was only $2.8 \%$. This is significantly lower than the amounts in the fibroblasts of the mother $(p=0.0180)$ and father $(p=0.0249)$ (Fig. 4c). These results show that DNA replication is impaired in fibroblasts of affected individuals.

As the principal phenotype in the individuals is neurological, we next assessed cell proliferation in the brain of dtymk mutant zebrafish larvae at 2 dpf using phospho-Ser10 Histone $\mathrm{H} 3 \mathrm{(pH} 3)$ staining. Histone $\mathrm{H} 3$ is phosphorylated at Ser10 by cyclin dependent kinase 1 in late G2 phase and mitosis and is a frequently used marker for cell proliferation. The number of proliferating cells in the forebrain of dtymk mutant and sibling embryos at 2dpf was evaluated (Fig. 4d). Quantification was limited to the forebrain as this is the homologous structure to the human cerebral cortex, which is most affected in the affected individual. As the larvae are morphologically undistinguishable at $2 \mathrm{dpf}$, the number of proliferating cells in the forebrain of individual embryos was counted and embryos were subsequently genotyped. We found the number of $\mathrm{pH} 3$-positive cells in the forebrain of dtymk mutant embryos to be significantly lower than the number of proliferating cells in wildtype $(p=0.0008)$ and heterozygous ( $p=0.0004)$ dtymk embryos (Fig. 4d). When comparing the sibling embryos, there is no difference in the number of proliferating cells in wildtype versus heterozygous dtymk embryos. In summary, we find severe defects in DNA replication in individual I-derived fibroblasts and in proliferation in the brain of dtymk mutant zebrafish.

As the biosynthesis of one of the nucleotides for DNA synthesis and repair is impaired, one could expect nucleotide pool imbalance in mutant fibroblasts and zebrafish. One of the most common consequences of nucleotide imbalance is the incorporation of ribonucleotides into genomic DNA [36]. Ribonucleotides differ from deoxyribonucleotides by the presence of a single reactive hydroxyl group at the 2' position of the ribose sugar, making RNA more susceptible to spontaneous hydrolysis [20]. The presence of ribonucleotides in genomic DNA is not favorable as they can impair DNA replication and render DNA more sensitive to strand breakage, leading to genome instability. We evaluated the presence of ribonucleotides in the genomic DNA by alkaline hydrolysis and subsequent alkaline gel electrophoresis (Fig. 5a, b). These experiments show that the genomic DNA isolated from dtymk -/- embryos migrates at a lower position and is more fragmented, indicated by a broader smear, in an alkaline agarose gel (Fig. 5a). This indicates a marked increased sensitivity of the genomic DNA of dtymk -/- embryos to alkaline hydrolysis reflective of elevated incorporation of ribonucleotides. When compared to the DNA from cells from Rnaseh2 -/- mice, a mutant strain known to incorporate high amounts of ribonucleotides in the genome [6, 28], the genomic DNA of dtymk -/- zebrafish shows a similar degree of fragility resulting in a broad smear of lower molecular weight and lower intensity (Supplementary Fig. 3c, online resource).

Virtually all DNA repair pathways involve some degree of repair-associated DNA synthesis. The effects of DTYMK deficiency on the process of DNA damage signaling was evaluated in vivo. Embryos were UV-irradiated at $24 \mathrm{hpf}$. DNA breakage was visualized by $\gamma \mathrm{H} 2 \mathrm{AX}$ staining. The embryos were allowed to recover for $24 \mathrm{~h}$ and the number of $\gamma \mathrm{H} 2 \mathrm{AX}$-positive cells, visible as small black dots throughout the embryo, in dtymk mutant fish was evaluated (Fig. 5c, d). From a batch of embryos from a heterozygous incross, two distinct populations can be discriminated: one group with high amounts of strongly stained $\gamma \mathrm{H} 2 \mathrm{AX}$ cells and another group with no to very low levels of $\gamma \mathrm{H} 2 \mathrm{AX}$-positive cells. The fraction of embryos from a dtymk heterozygous incross with high amounts of $\gamma \mathrm{H} 2 \mathrm{AX}$-positive cells was about $25 \%$. Genotyping confirmed that these were the dtymk homozygous mutant fraction. This analysis shows elevated levels of $\gamma \mathrm{H} 2 \mathrm{AX}$ persisting in the dtymk mutant embryos $24 \mathrm{~h}$ post UV-irradiation compared to the wild-type (sib) counterparts. This abnormally elevated DNA damage response signaling 

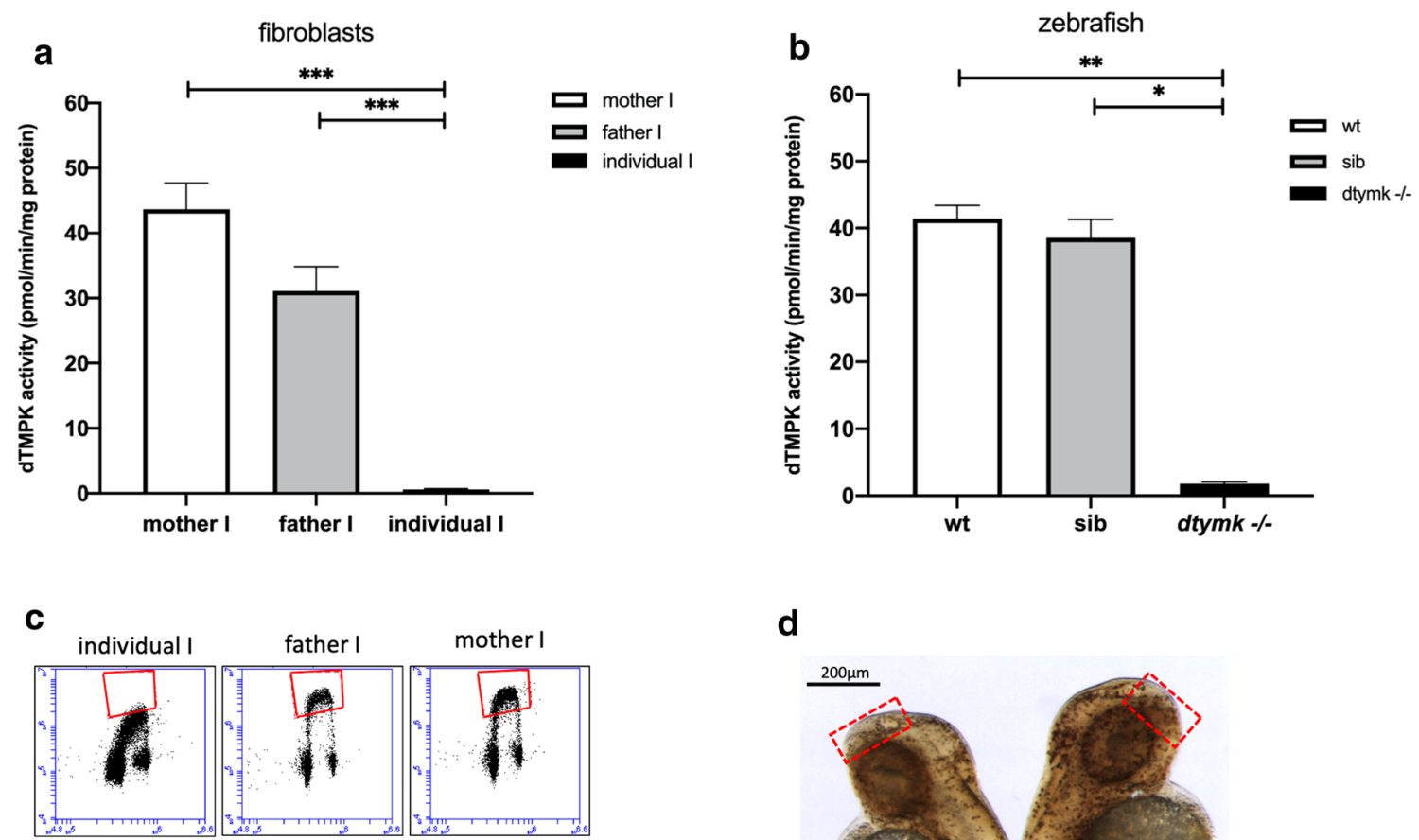

d
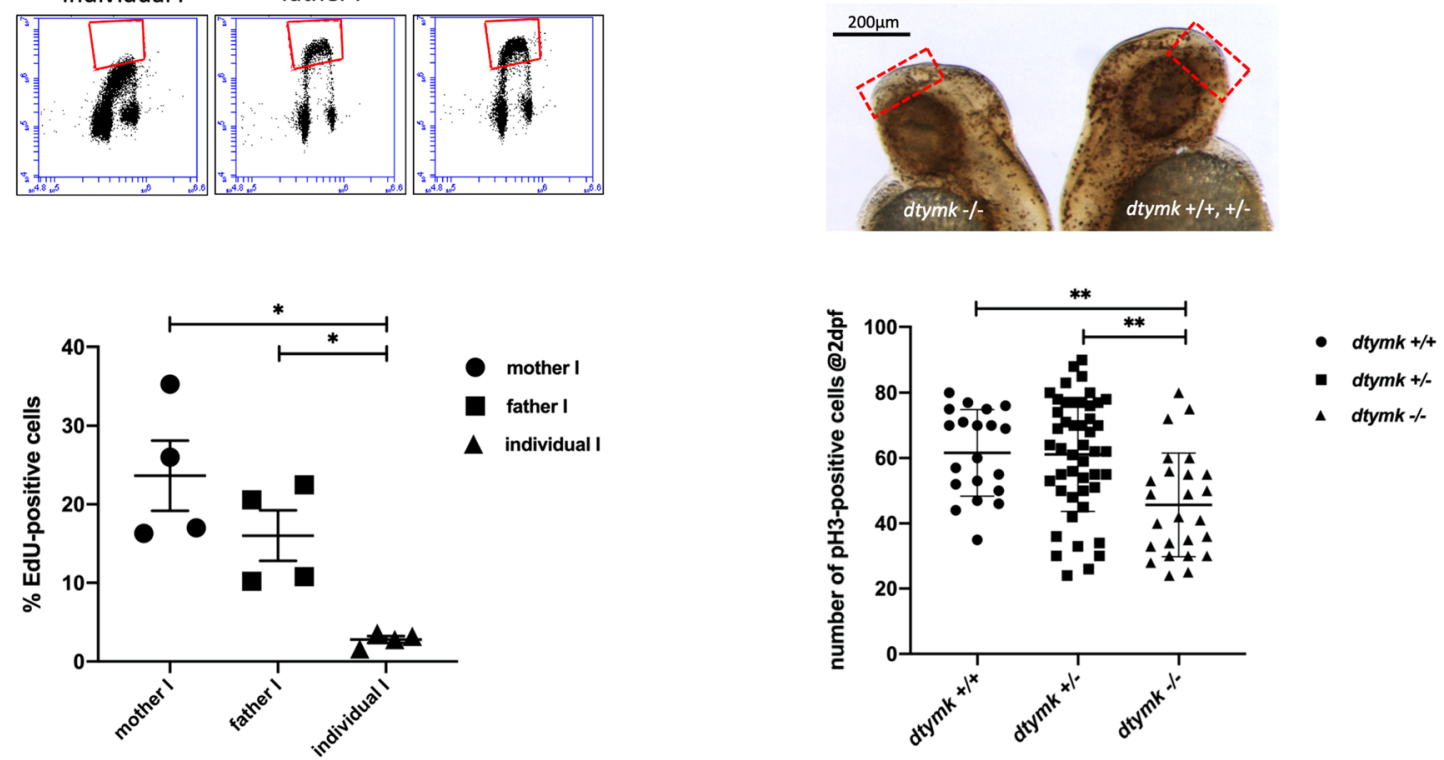

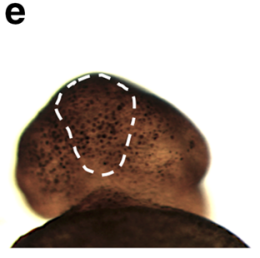

dtymk -/-

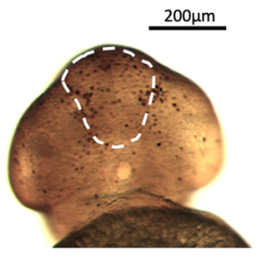

sib is consistent with elevated levels of unrepaired DNA breaks in the dtymk mutants under these conditions (Fig. 5c, d).

In a recent study, DTYMK was suggested to cause mitochondrial DNA (mtDNA) depletion [29]. Therefore, we studied the mitochondrial genome and function in cultured fibroblasts of individual I (Fig. 6) and the parents of individual I. For additional reference, we included 5 fibroblast samples of healthy controls. The mtDNA copy number in fibroblasts of individual I was found to be lower than that of the parents but this was not statistically different. 
४Fig. 4 dTMPK activity and replication defects in fibroblasts and zebrafish. a dTMPK activity measured in fibroblasts of family I. In both parents, activity was readily detected and was lower in the fibroblasts of the father. dTMPK activity was hardly detectable in individual I $(n=6)$. Mother vs. individual I $\left(* * *, p=1.46 \times 10^{-6}\right.$, unequal variance). Father vs. individual I $\left(* * *, p=5.77 \times 10^{-6}\right.$, unequal variance). b dtmpk activity in lysates of zebrafish at $5 \mathrm{dpf}$. Lysates were prepared from pools of 35-50 embryos of indicated genotypes. $n=2-3$ and measurements were performed 2 times. dtmpk activity was negligible in mutant embryos and robustly detected in both sibling embryos as embryos of the same age from an unrelated wildtype strain (AB). Wt vs. mut: **, $p=0.0001)$; Sib vs. mut: *, $p=0.0028$. c EdU incorporation in the fibroblasts of family I. Representative flow cytometry profiles are shown from fibroblasts of the individual and the parents. The red boxes denote the EdU positive (S-phase) cell populations. Quantification of the percentage of EdU-positive (S-phase) cells shows that the fibroblasts of individual I have significantly fewer proliferating cells when compared to the fibroblasts of the mother $(*, p=0.0180$, unequal variance) and father $(*, p=0.0249$, unequal variance). Indicated values are average $\%$ of EdU-positive cells $\pm \mathrm{SEM}, n=4)$. d Whole-mount $\mathrm{pH} 3$-immunostaining showing proliferating cells in wildtype $($ dtymk $+/+)$, heterozygous (dtymk+/-) and dtymk-knockout (dtymk -/-) embryos at 2dpf. Representative images of side-view of an embryo with many proliferating cells (black dots, right image) and an embryo with less proliferating cells (left image). The red, dotted rectangle represents the area that was analyzed for the quantification of the number of proliferating cells in the forebrain. The scale bar represents $200 \mu \mathrm{m}$. Quantification of the number of $\mathrm{pH} 3$-positive cells in the brain indicates that the mutant embryos have significantly less $\mathrm{pH} 3$-positive cells in the brain when compared to wildtype (**, $p=0.0008$ ) and heterozygous (**, $p=0.0004$ ) embryos, $n=9-24$ embryos per group, two biological replicates. Indicated values are average \pm SEM. e Whole-mount TUNEL staining showing apoptotic cells in sibling (sib: dtymk+/+or dtymk+/-) and dtymk-knockout (dtymk -/-) embryos at 2dpf. Representative frontal images of an embryo with many apoptotic cells (black dots, left image, dtymk -/-) and an embryo with less apoptotic cells (right image, sib). The white, dashed area represents the forebrain, the area that was analyzed for the quantification of the number of apoptotic cells in the forebrain. Embryos were genotyped after staining and imaging. The scale bar represents $200 \mu \mathrm{m}$. f Quantification of the number of apoptotic cells in the forebrain in wildtype, heterozygous and dtymk-knockout embryos at $2 \mathrm{dpf}$. The mutant embryos have significantly more $(* * *, p<0.0001)$ apoptotic cells in the forebrain when compared to wildtype $\left(* * *, p=6.45 \times 10^{-6}\right.$, equal variance) and heterozygous (***, $p=7.54 \times 10^{-6}$, unequal variance) embryos, $n=23-46$ embryos per group, two biological replicates. Indicated values are average \pm standard deviation

Moreover, mtDNA copy number in fibroblasts of the parents of individual $\mathrm{I}$ is higher than in control fibroblasts (Fig. 6a) and the mtDNA copy number of individual $I$ is in the same range as the control cells.

Deficiencies in the replication machinery of the mtDNA can also result in deletions in the mtDNA. Therefore, we studied the integrity of the mitochondrial genome by means of long-range PCR, amplifying the complete mitochondrial genome (Fig. 6b). The mitochondrial genome amplified from individual I and its parents migrate at the expected length of 16,5 kilobases and show no indications of mtDNA deletions. We conclude that the mtDNA copy number and integrity are not compromised by DTYMK deficiency. Finally, mitochondrial function was assessed. We evaluated enzyme activity of respiratory complexes I-V using a spectrophotometric assay as used in mitochondrial disease diagnostics (Fig. 6c). individual I shows no significant alterations in the enzyme activities of complexes $\mathrm{I}-\mathrm{V}$, indicating that mitochondrial respiratory function in individual I is comparable to that of the parents. In conclusion, we find no significant indications of mitochondrial dysfunction in fibroblasts of individual I.

\section{Discussion}

We report loss-of-function of DTYMK as the genetic cause of a novel, severe neurodegenerative disease in two unrelated individuals with a dramatic neurodevelopmental decline. Brain pathology confirmed massive neuronal dropout in virtually the entire brain, sparing the brain stem. This striking neurodegenerative phenotype is also observed in dtymk mutant zebrafish which exhibit microcephaly, brain edema and neuronal apoptosis (Fig. 3). These results, as well as the cerebral atrophy that only sets in postnatally and the presence of apoptotic cells in the brain of individual I (Fig. 2j) implicate neuronal apoptosis as one of the hallmarks of this disease.

This report is the second study describing DTYMK variants in the context of human disease. Lam et al. recently reported two brothers with microcephaly, hypotonia and severe intellectual disability showing compound heterozygous variants in DTYMK [17]. They predicted mitochondrial depletion in one of the affected individuals, but functional data to support a mitochondrial defect was not provided [17]. In the current study, we present DTYMK variants in two unrelated families and provide biochemical data from fibroblasts of affected individuals and an additional animal model. Furthermore, we demonstrate important aspects of the pathogenic mechanism. Although the clinical phenotype of our individuals highly resembles that described previously, we could not document mtDNA depletion. (Fig. 6a). We acknowledge that measurement of mtDNA copy number in fibroblasts (or peripheral blood) is not representative for the copy number in affected tissues, as compensatory mechanisms can mask mtDNA depletion $[9,30]$. For diagnostic purposes, mtDNA copy number can only be reliably assessed in a muscle biopsy of affected individuals. Unfortunately, we do not have access to muscle tissue of the described individuals. Still, additional functional studies failed to demonstrate mitochondrial dysfunction. Mitochondrial parameters, mtDNA integrity and respiratory chain activity, were all within the normal range in individual I. Also, all biochemical indicators for mitochondrial dysfunction from our individuals that were measured in serum and 


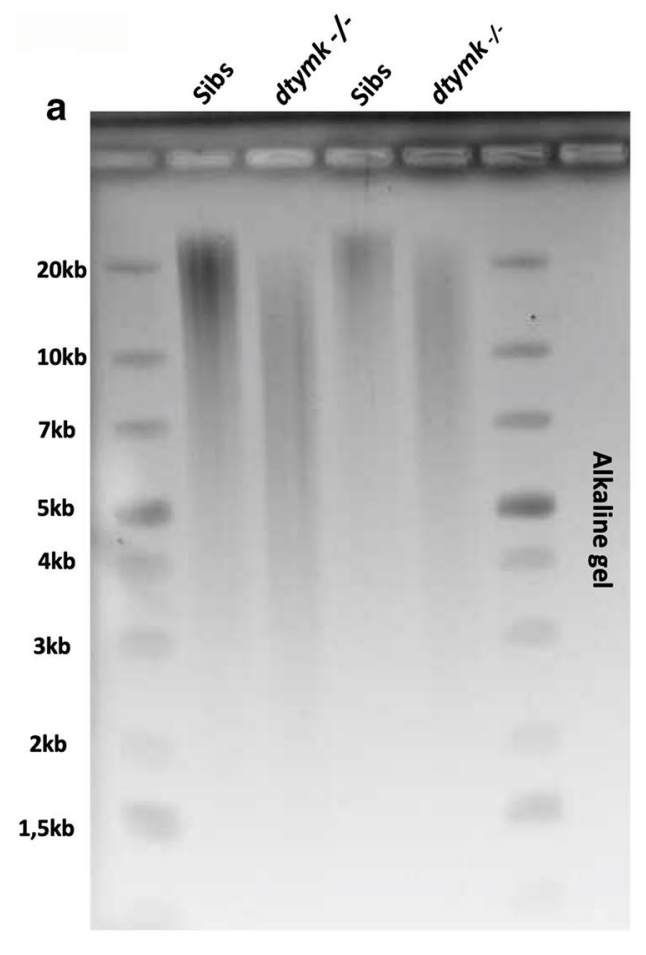

b
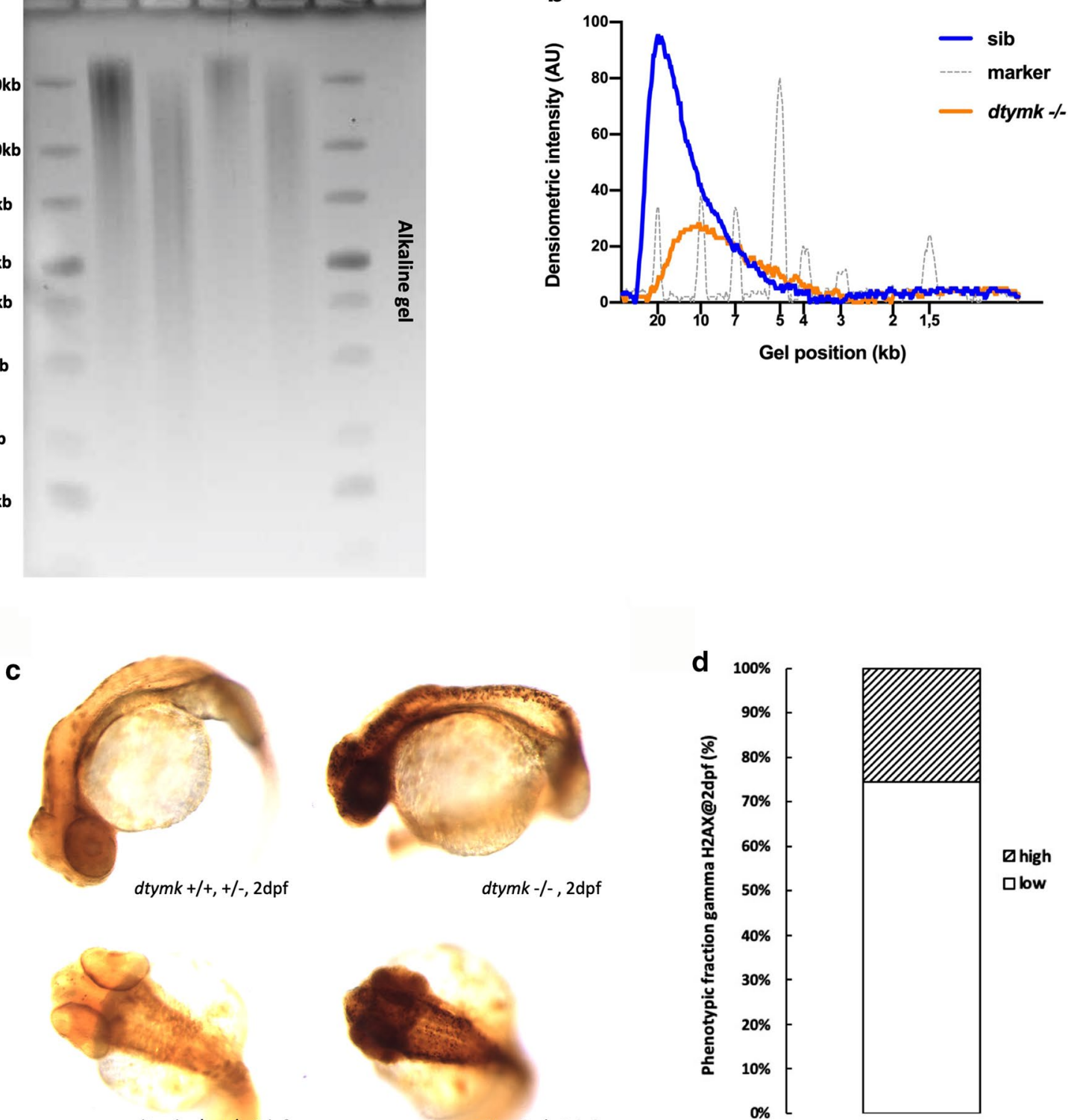

Dhigh

口low
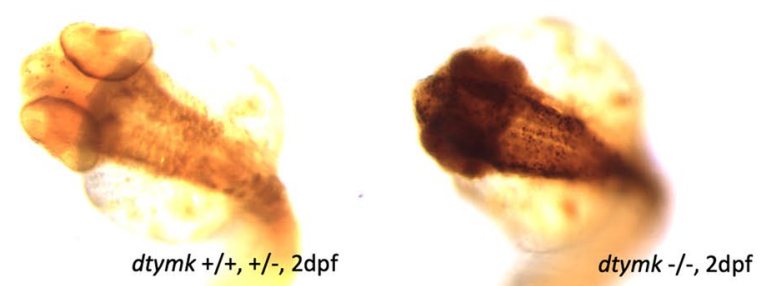

catalyses the reduction of ribonucleoside diphosphates to deoxyribonucleoside diphosphates in the de novo biosynthetic pathways of all four canonical dNTP. Another human neurodegenerative disease, linked to dTTP metabolism and caused by pathogenic variants in $C A D$ (Fig. 1a) is developmental and epileptic encephalopathy 50 (DEE50) [15, 22]. This is an autosomal recessive, progressive neurodegenerative neurometabolic disorder characterized by delayed psychomotor development, early onset seizures, severe developmental regression, and normocytic anemia. Onset is within the first months or years of life $[15,22]$. Lastly, DTYMK deficiency resembles Aicardi-Goutières syndrome (AGS), 
४Fig. 5 Ribonucleotide incorporation and DNA damage signaling. a Representative image of alkaline gel electrophoresis of genomic DNA. Genomic DNA of dtymk mutant embryos (dtymk -/-) and sibling (sibs) embryos at 5dpf was subjected to alkaline gel electrophoresis. The first two lanes contain $5 \mu \mathrm{g}$ of genomic DNA of sibling (Sibs) and dtymk homozygous mutant (dtymk -/-) embryos, respectively. The following two lanes contain $2.5 \mu \mathrm{g}$ of genomic DNA of sibling (Sibs) and dtymk homozygous mutant (dtymk -/-) embryos, respectively. The first and last lane contain the marker Generuler $1 \mathrm{~kb}$ Plus DNA ladder (Thermo Fisher). Three biological replicates produced highly similar results. b Quantification of alkaline gel electrophoresis. Densiometric intensity values of a line drawn from the slot to the bottom of the gel were determined using ImageJ and plotted as a function of distance in the gel. The genomic DNA of homozygous mutant (dtymk -/) embryos, marked in orange, migrates at a lower molecular weight as the DNA of sibling embryos, marked in blue. Results indicate an increased sensitivity to alkaline hydrolysis of the genomic DNA of homozygous dtymk mutant (dtymk -/-) embryos. The position of the molecular weight marker is indicated by the grey line and its respective peaks corresponding to different molecular weights are indicated in the $x$-axis (gel position). c Representative images of side view (upper panels) and dorsal view (lower panels) of embryos at 2dpf showing sites of DNA-damage, visualized by $\gamma \mathrm{H} 2 \mathrm{AX}$-staining (black dots). DNA damage was induced by UV-irradiation at $24 \mathrm{hpf}$ followed by a recovery time of $24 \mathrm{~h}$. The left panels show sibling embryos (dtymk $+/+,+/-)$ and the right panels show mutant (dtymk -/-) embryos. The mutant embryos clearly show many sites of unrepaired DNA-damage, whereas the sibling embryos hardly show any sites of DNA-damage. Embryos were imaged using identical camera and illumination settings. The scale bar represents $200 \mu \mathrm{m}$. d Quantification of the phenotypic fraction of embryos at $2 \mathrm{dpf}$ that show a high amount (high) vs. a low amount (low) of sites of DNA damage. The phenotypic fraction is consistent with the expected fraction of $25 \%$ as expected from a heterozygous incross of heterozygous dtymk fish. The genotype of the low vs. high phenotypic fraction was verified by Sanger sequencing. The number of embryos included in the analysis is $n=13$ for embryos with high amounts of staining (dtymk mutants) and $n=38$ for sibling embryos showing low to no $\gamma \mathrm{H} 2 \mathrm{AX}$-staining. Two biological replicates produced similar results

both at the clinical as at the pathophysiological level. AGS is characterized by progressive microcephaly, psychomotor retardation and often death in early childhood [8]. White matter destruction and brain atrophy are shared phenotypic features of AGS and DTYMK deficiency. Clinical differences are that in AGS, intracranial calcification and systemic features (thrombocytopenia, hepatosplenomegaly and cerebrospinal fluid lymphocytosis) are present [2]. Importantly, at the mechanistic level, AGS and DTYMK deficiency are similar as both are characterized by elevated ribonucleotide incorporation in the genome. In AGS, this is explained by pathogenic variants in the genes encoding ribonuclease $\mathrm{H} 2$ subunits [8]. Ribonuclease $\mathrm{H} 2$ is the key enzyme, involved in ribonucleotide excision repair (RER). Impairment of this pathway results in the increased genomic incorporation of ribonucleotides [8]. Similarly, in DTYMK deficiency, ribonucleotide incorporation is explained by the lack of sufficient postnatal availability of one of the building blocks of
DNA, due to a complete block of the biosynthetic pathway of dTTP.

A notable difference between the human phenotype and the dtymk-deficient zebrafish model is the degree in which eye development is affected. In both individuals, some degree of visual impairment was noted as the individuals showed no visual tracking of objects and were thought only to be able to distinguish light and dark. However, the eyes were morphologically normal in both individuals. In the zebrafish model, there is a severe eye phenotype consisting of smaller, deformed eyes lacking multiple cellular layers (Supplementary Fig. 2d, online resource). Similar eye phenotypes have been reported in knockout models of other enzymes of nucleotide metabolism [38]. In perplexed mutants, which have a pathogenic variant in cad (carbamoylphosphate synthase 2 , aspartate transcarbamylase and dihydroorotase), the enzyme active in the initial step of de novo dTTP synthesis (Fig. 1a), eye defects are similar to dtymkdeficient embryos: eyes are small and lack retinal lamination (Supplementary Fig. 2d, online resource) [38]. Other phenotypic features of perplexed mutants include absence of the lower jaw cartilage. This cartilage defect is also observed in dtymk mutants (Fig. 3c). A second mutant affecting de novo synthesis is hi688, which is defective in ribonucleotide reductase R2 ( $\mathrm{rrm} 2$ ), is early lethal and leads to a general degeneration phenotype $[11,38]$. This phenotype is also reminiscent of the dtymk phenotype in which early lethality and general degeneration is observed.

We provide additional insight into the phenotypic presentation of severely compromised dTMPK activity. We show a marked impairment in DNA replication in fibroblasts of an affected individual (Fig. 4c). In addition, we demonstrated significantly reduced cellular proliferation (Fig. 4d) and apoptosis (Fig. 4e, f) in the brain of dtymk mutant zebrafish and elevated levels of UV-induced $\gamma \mathrm{H} 2 \mathrm{AX}$ suggestive of elevated genomic instability in zebrafish embryos (Fig. 5c, d). Genome instability is most probably caused by ribonucleotide incorporation in the genome (Fig. 5a, b). Ribonucleotide incorporation is one of the most abundant forms of DNA damage in eukaryotic cells, making the DNA more fragile $[21,23,36]$. Therefore, a repair mechanism: RER, is in place to remove ribonucleotides from genomic DNA. A critical component of RER is RNaseH2 [19]. Mouse mutants for $\mathrm{RNaseH} 2$ accumulate large numbers of ribonucleotides in their genomic DNA resulting in genome instability, arrest in cellular proliferation and a p53-dependent DNA-damage response $[6,28]$. We show that dtymk mutant zebrafish exhibit elevated incorporation of ribonucleotides in the genomic DNA (Fig. 5a, b). When compared to genomic DNA of Rnaseh2 -/- mice, the dtymk -/- genomic DNA shows as similar behavior on alkaline gels: a broad smear of lower molecular weight and reduced intensity due to increased degradation (Supplementary Fig. 3c, online 

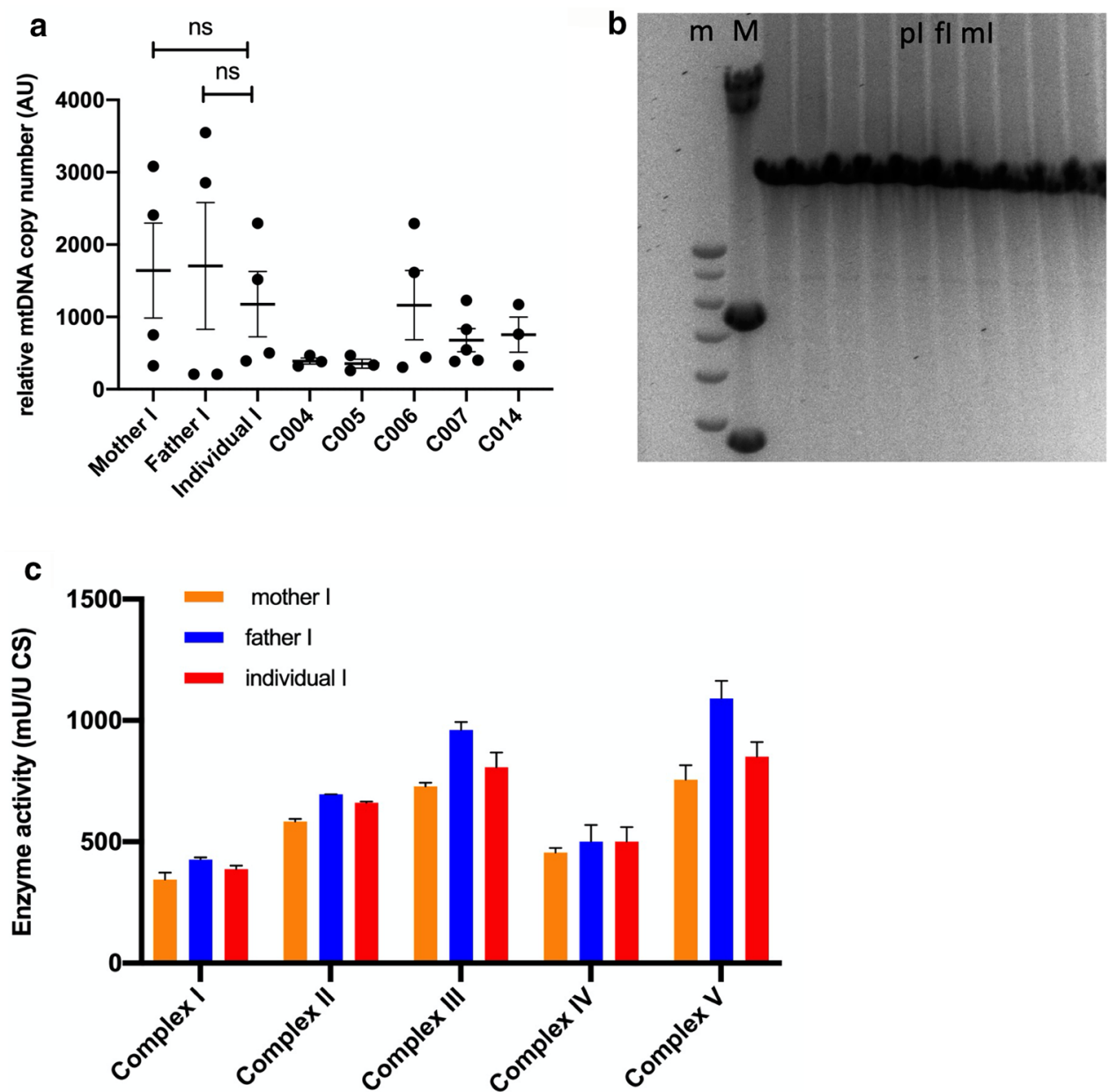

Fig. 6 Mitochondrial DNA copy number, integrity and complex activity. a Relative mtDNA copy number in fibroblasts of individual $\mathrm{I}$, the parents of individual I and 5 healthy controls. mtDNA copy number was assessed by comparing expression levels of a mitochondrial DNA fragment (D-loop) versus a nuclear encoded gene $(B 2 M)$. Relative mtDNA copy number was calculated by calculating $\triangle C T$ (=CT nuclear gene, B2M-CT mitochondrial gene, $D$-loop) and converting $\triangle C T$ to relative expression levels (relative expression $=2^{\Delta C T}$ ) [31]. Results are pooled values from 4 experiments and plotted as average \pm SEM. b MtDNA integrity by long-range PCR. Lane 1 and 2 contain DNA ladders (m); $1 \mathrm{~kb}$ Plus ladder and (M); HindIII-digested $\lambda$ DNA fragments ladder, respectively. Lanes 3-6 contain anonymized diagnostic samples, lane 7 contains the sample of individual I, lane 8 the father of individual I and lane 9 the mother of individual I. Lane 10-12 also contain anonymized diagnostic samples. This procedure is a diagnostic test, performed at Clinical Genetics at Maastricht $\mathrm{UMC}+$. No large mtDNA deletions could be observed. c Mitochondrial respiratory complex activity. Enzymatic activity of mitochondrial respiratory complexes I-V were measured using a spectrophotometer in lysates of cultured fibroblasts [33]. Relative values are calculated using the value of Citrate Synthase activity as the reference value. Bars are averages of 2 measurements. This assay is a diagnostic assay, performed at the Translational Metabolic Laboratory of Radboud UMC resource). The elevated accumulation of ribonucleotides in genomic DNA associated with impaired DNA replication, reduced cellular proliferation and elevated DNA damage response signaling represents a plausible explanation for the severe phenotypic presentation and the early embryonic lethality ( $40 \%$ death at $5 \mathrm{dpf}$ ) observed in dtymk mutant zebrafish. This scenario may contribute to the progressive loss of cerebral cortex tissue in the individuals carrying $D T Y M K$ variants $[1,34]$.

It is remarkable that an apparently complete DTYMK deficiency is compatible with life. Due to a complete block in the metabolic pathway for dTTP synthesis, an essential building block of DNA cannot be generated. There is no known alternative biochemical pathway that could provide an adequate supply of dTTP to sustain DNA replication and repair as both known pathways (de novo and salvage pathway) converge before the defect in DTYMK (Fig. 1a). This raises two outstanding questions: First, what is the compensation mechanism allowing fetal brain development and survival until birth without the ability to generate dTTP? Second, why is the brain most affected? One possibility is that the missense alleles described in the two families in this study still show minimal residual activity, sufficient for very limited DNA synthesis and repair. On the other hand, the 
zebrafish model is a frameshift allele giving rise to a grossly truncated protein with almost undetectable enzyme activity. The phenotypic similarity of this loss-of-function allele in the zebrafish to that of the affected individuals coupled to the fact that both models show almost undetectable dTMPK enzyme activity strongly suggest that the human variants are loss-of-function variants.

The fact that both in fibroblast as in zebrafish models, dTTP nucleotide levels resemble those of normal controls (Supplementary Fig. 3a, b, online resource) together with the fact that both affected individuals and the homozygous zebrafish survive beyond birth could suggest the intriguing possibility of a compensatory pathway for dTTP generation. A prima facie candidate enzyme would be TMPK2 (or UMP-CMP kinase 2, CMPK2) [7, 39]. However, as this enzyme has no apparent capability to use dTMP as a substrate $[5,7,39]$ it would appear unlikely that CMPK2 can fulfill this function. Thus, a compensatory pathway for dTTP generation remains to be proven.

It is known that imbalanced dNTP pools cause replication stress that can in turn trigger neuronal apoptosis [25]. Widespread apoptosis is what was observed in the brains of our affected individuals, and in the zebrafish. Why neurons are particularly vulnerable to this imbalance is not clear at this time. A restricted expression pattern of $D T Y M K$ is unlikely to be the answer as $D T Y M K$ is expressed in all human tissues. Cerebral cortex, hippocampus and caudate nucleus show medium expression, while cerebellum has high protein expression (https://www.prote inatlas.org/ENSG00000168393-DTYMK/tissue). Interestingly, expression in brain mouse embryo (E15) (http:// www.informatics.jax.org/marker/MGI:108396) shows highest Dtymk expression levels in the forebrain, moderate levels in the midbrain (tectum) and low expression in the hindbrain. This might provide a clue to why the forebrainderived structures (cerebral cortex and basal ganglia) are most affected in the described individuals. Alternatively, a restricted expression pattern of a putative compensatory enzyme could explain the vulnerability of the brain in this disease.

In conclusion, we show that novel germline variants in DTYMK cause profound postnatal neurodegeneration and marked developmental arrest. Our studies directly link thymidine biosynthesis to genome integrity and postnatal neuronal viability.

Supplementary Information The online version contains supplementary material available at https://doi.org/10.1007/s00401-021-02394-0.

Acknowledgements We thank the families involved for their extensive collaboration. Rick Kamps is acknowledged for expert help in histology and Yvette E.G. Barrois for additional genotyping. We thank Alexandra Hendrickx for providing mtDNA diagnostics and Martin Reijns and Andrew Jackson (University of Edinburgh) for kindly providing
Rnaseh2 -/- cells. This project was funded by Metakids grant number 2018-081 (Metakids.nl), awarded to JV.

\section{Declarations}

Conflict of interest The authors declare no competing interests.

Open Access This article is licensed under a Creative Commons Attribution 4.0 International License, which permits use, sharing, adaptation, distribution and reproduction in any medium or format, as long as you give appropriate credit to the original author(s) and the source, provide a link to the Creative Commons licence, and indicate if changes were made. The images or other third party material in this article are included in the article's Creative Commons licence, unless indicated otherwise in a credit line to the material. If material is not included in the article's Creative Commons licence and your intended use is not permitted by statutory regulation or exceeds the permitted use, you will need to obtain permission directly from the copyright holder. To view a copy of this licence, visit http://creativecommons.org/licenses/by/4.0/.

\section{References}

1. Alcantara D, O'Driscoll M (2014) Congenital microcephaly. Am J Med Genet C Semin Med Genet 66C:124-139

2. Ali M, Highet LJ, Lacombe D et al (2006) A second locus for Aicardi-Goutieres syndrome at chromosome 13q14-21. J Med Genet 43:444-450

3. Arnér ES, Eriksson S (1995) Mammalian deoxyribonucleoside kinases. Pharmacol Ther 67:155-186

4. Bourdon A, Minai L, Serr V et al (2007) Mutation of RRM2B, encoding p53-controlled ribonucleotide reductase (p53R2), causes severe mitochondrial DNA depletion. Nat Genet 39:776-780

5. Carnrot C, Wehelie R, Eriksson S et al (2003) Molecular characterization of thymidine kinase from Ureaplasma urealyticum: nucleoside analogues as potent inhibitors of mycoplasma growth. Mol Microbiol 50:771-780

6. Cerritelli SM, Crouch RJ (2016) The balancing act of ribonucleotides in DNA. Trends Biochem Sci 41:434-445

7. Chen YL, Lin DW, Chang ZF (2008) Identification of a putative human mitochondrial thymidine monophosphate kinase associated with monocytic/macrophage terminal differentiation. Genes Cells 13:679-689

8. Crow YJ, Leitch A, Hayward BE et al (2006) Mutations in genes encoding ribonuclease $\mathrm{H} 2$ subunits cause Aicardi-Goutières syndrome and mimic congenital viral brain infection. Nat Genet 38:910-916

9. Frangini M, Rampazzo C, Franzolin E, Lara MC, Vilà MR, Martí $R$ et al (2009) Unchanged thymidine triphosphate pools and thymidine metabolism in two lines of thymidine kinase 2-mutated fibroblasts. FEBS J 276:1104-1113

10. Gagnon JA, Valen E, Thyme SB et al (2014) Efficient mutagenesis by Cas 9 protein-mediated oligonucleotide insertion and largescale assessment of single-guide RNAs. PLoS ONE 9(5):e98186

11. Golling G, Amsterdam A, Sun Z et al (2002) Insertional mutagenesis in zebrafish rapidly identifies genes essential for early vertebrate development. Nat Genet 31:135-140

12. González-Vioque E, Torres-Torronteras J, Andreu AL et al (2011) Limited dCTP availability accounts for mitochondrial DNA depletion in mitochondrial neurogastrointestinal encephalomyopathy (MNGIE). PLoS Genet 7:e1002035

13. Huang SH, Tang A, Drisco B, Zhang SQ et al (1994) Human dTMP kinase: gene expression and enzymatic activity coinciding 
with cell cycle progression and cell growth. DNA Cell Biol 13:461-471

14. Kevelam SH, Bugiani M, Salomons GS, Feigenbaum A, Blaser S, Prasad C et al (2013) Exome sequencing reveals mutated SLC19A3 in patients with an early-infantile, lethal encephalopathy. Brain 136:1534-1543

15. Koch J, Mayr JA, Alhaddad B, Rauscher C, Bierau J, KovacsNagy R et al (2017) CAD mutations and uridine-responsive epileptic encephalopathy. Brain 140:279-286

16. Labun K, Montague TG, Gagnon JA et al (2016) CHOPCHOP v2: a web tool for the next generation of CRISPR genome engineering. Nucleic Acids Res 44:W272-W276

17. Lam CW, Yeung WL, Ling TK et al (2019) Deoxythymidylate kinase, DTYMK, is a novel gene for mitochondrial DNA depletion syndrome. Clin Chim Acta 496:93-99

18. Lawrence $C$ (2007) The husbandry of zebrafish (Danio rerio): a review. Aquaculture 269:1-20

19. Lindsey-Boltz LA, Kemp MG, Hu J et al (2015) Analysis of ribonucleotide removal from DNA by human nucleotide excision repair. J Biol Chem 290:29801-29807

20. Li YF, Breaker RR (1999) Kinetics of RNA degradation by specific base catalysts of transesterification. J Am Chem Soc 121:5364-5372

21. Meroni A, Mentegari E, Crespan E et al (2017) The incorporation of ribonucleotides induces structural and conformational changes in DNA. Biophys J 113:1373-1382

22. Ng BG, Wolfe LA, Ichikawa M, Markello T, He M, Tifft CJ et al (2015) Biallelic mutations in CAD, impair de novo pyrimidine biosynthesis and decrease glycosylation precursors. Hum Mol Genet 24:3050-3057

23. Nick McElhinny SA, Kumar D, Clark AB, Watt DL, Watts BE, Lundström E-B et al (2010) Genome instability due to ribonucleotide incorporation into DNA. Nat Chem Biol 6:774-781

24. Nishino I, Spinazzola A, Hirano M (1999) Thymidine phosphorylase gene mutations in MNGIE, a human mitochondrial disorder. Science 283:689-692

25. Oliver FJ, Collins MK, López-Rivas A (1996) dNTP pool imbalance as a signal to initiate apoptosis. Experientia 52:995-1000

26. Ostermann N, Schlichting I, Brundiers R, Konrad M, Reinstein $\mathrm{J}$, Veit $\mathrm{T}$ et al (2000) Insights into the phosphoryltransfer mechanism of human thymidylate kinase gained from crystal structures of enzyme complexes along the reaction coordinate. Structure 8:629-642

27. Rahn JJ, Bestman JE, Stackley KD, Chan SS (2015) Zebrafish lacking functional DNA polymerase gamma survive to juvenile stage, despite rapid and sustained mitochondrial DNA depletion, altered energetics and growth. Nucleic Acids Res 43:10338-10352

28. Reijns MA, Rabe B, Rigby RE et al (2012) Enzymatic removal of ribonucleotides from DNA is essential for mammalian genome integrity and development. Cell 149:1008-1022

29. Rodenburg RJT (2011) Biochemical diagnosis of mitochondrial disorders. J Inherit Metab Dis 34:283-292

30. Saada A (2008) Mitochondrial deoxyribonucleotide pools in deoxyguanosine kinase deficiency. Mol Genet Metab 95:169-173

31. Sambrook J, Russell DW (2016) Alkaline agarose gel electrophoresis. CSH Protoc. https://doi.org/10.1101/pdb.prot4027

32. Stankiewicz P, Khan TN, Szafranski P et al (2017) Haploinsufficiency of the chromatin remodeler BPTF causes syndromic developmental and speech delay, postnatal microcephaly, and dysmorphic features. Am J Hum Genet 101:503-515

33. Talbot JC, Amacher SL (2014) A streamlined CRISPR pipeline to reliably generate zebrafish frameshifting alleles. Zebrafish 11:583-585

34. Uehara R, Cerritelli SM, Hasin N et al (2018) Two RNase H2 mutants with differential rNMP processing activity reveal a threshold of ribonucleotide tolerance for embryonic development. Cell Rep 25:1135-1145

35. Venselaar H, Te Beek TAH, Kuipers RKP, Hekkelman ML, Vriend G (2010) Protein structure analysis of mutations causing inheritable diseases. An e-Science approach with life scientist friendly interfaces. BMC Bioinform 11:548

36. Wallace BD, Williams RS (2014) Ribonucleotide triggered DNA damage and RNA-DNA damage responses. RNA Biol 11:1340-1346

37. Wang L (2007) The role of Ureaplasma nucleoside monophosphate kinases in the synthesis of nucleoside triphosphates. FEBS J 274:1983-1990

38. Willer GB, Lee VM, Gregg RG et al (2005) Analysis of the Zebrafish perplexed mutation reveals tissue-specific roles for de novo pyrimidine synthesis during development. Genetics 170:1827-1837

39. Xu Y, Johansson M, Karlsson A (2008) Human UMP-CMP kinase 2, a novel nucleoside monophosphate kinase localized in mitochondria. J Biol Chem 283:1563-1571

Publisher's Note Springer Nature remains neutral with regard to jurisdictional claims in published maps and institutional affiliations. 